\title{
A Novel Picture Fuzzy $n$-Banach Space with Some New Contractive Conditions and Their Fixed Point Results
}

\author{
Awais Asif, ${ }^{1}$ Hassen Aydi $\mathbb{D}^{2,3,4}$ Muhammad Arshad, ${ }^{1}$ and Zeeshan Ali ${ }^{1}$ \\ ${ }^{1}$ Department of Math \& Stats, International Islamic University Islamabad, Pakistan \\ ${ }^{2}$ Nonlinear Analysis Research Group, Ton Duc Thang University, Ho Chi Minh City, Vietnam \\ ${ }^{3}$ Faculty of Mathematics and Statistics, Ton Duc Thang University, Ho Chi Minh City, Vietnam \\ ${ }^{4}$ China Medical University Hospital, China Medical University, Taichung 40402, Taiwan \\ Correspondence should be addressed to Hassen Aydi; hassen.aydi@tdtu.edu.vn
}

Received 23 July 2020; Revised 2 September 2020; Accepted 4 September 2020; Published 25 September 2020

Academic Editor: Serkan Araci

Copyright (c) 2020 Awais Asif et al. This is an open access article distributed under the Creative Commons Attribution License, which permits unrestricted use, distribution, and reproduction in any medium, provided the original work is properly cited.

\begin{abstract}
A picture fuzzy $n$-normed linear space $\left(N_{\mathrm{PF}}\right)$, a mixture of a picture fuzzy set and an $n$-normed linear space, is a proficient concept to cope with uncertain and unpredictable real-life problems. The purpose of this manuscript is to present some novel contractive conditions based on $N_{\mathrm{PF}}$. By using these contractive conditions, we explore some fixed point theorems in a picture fuzzy $n$-Banach space $\left(B_{\mathrm{PF}}\right)$. The discussed modified results are more general than those in the existing literature which are based on an intuitionistic fuzzy $n$-Banach space $\left(B_{\mathrm{IF}}\right)$ and a fuzzy $n$-Banach space. To express the reliability and effectiveness of the main results, we present several examples to support our main theorems.
\end{abstract}

\section{Introduction}

In various real-life problems, for a suitable mapping, the existence of a solution and existence of a fixed point (FP) are equivalent. Thus, the existence of a FP is a proficient technique to cope with awkward and difficult problems in real-life issues. Various scholars have utilized such results in the environment of many fields $[1,2]$. The extensive useful techniques capable with both algebraic and topological properties are those of a normed linear space (NLS), but the continuous maps are more proficient in the sense of NLS. Moreover, in a metric space, every contractive map is uniformly continuous. One of the fundamental applications of Banach's contraction principle is the "Picard's theorem," which is the basic theorem for the existence and uniqueness of solution to the ordinary differential equations. Various scholars have utilized this application in the environment of a partial differential equation [3], in the Gauss-Seidel method for evaluating systems of linear equations [4], in the proof of the inverse function theorem [5], etc.
The theory of a fuzzy set (FS) was investigated by Zadeh [6], characterized by only positive grades restricted to $[0,1]$. FS has achieved more success due to its ability to cope with complications and troubles. However, in some practice cases, the concept of FS cannot cope with complications and uncertainty because of lack of knowledge of the problem. Therefore, Atanassov [7] investigated the intuitionistic FS (IFS) containing both positive and negative grades, whose sum is bounded to $[0,1]$. IFS is regarded as a more improved way to cope with complex and awkward information. Further, Cường [8] investigated the picture FS (PFS) including positive, abstinence, and negative grades, whose sum is bounded to $[0,1]$. PFS is regarded as a more improved way to deal with even more complex information. For more related works, we may refer to References [9-16].

Keeping the advantages of the PFS, the objective of this manuscript is summarized in the following ways:

(1) To present some novel contractive conditions, we used $N_{\mathrm{PF}}$ as a basis 
(2) By using these contractive conditions, some fixed point theorems are explored for a picture fuzzy $n$-Banach space $\left(B_{\mathrm{PF}}\right)$. These results are more modified and more general than the existing results which are based on an intuitionistic fuzzy $n$-Banach space $\left(B_{\mathrm{IF}}\right)$ and a fuzzy $n$-Banach space

(3) To express the reliability and effectiveness of the explored approaches, we explain examples in support of the main results

The rest of this manuscript is summarized in the following ways: In Section 2, we review some basic notions like $N_{\text {IF }}$ and their related properties used in the presented work. In Section 3, we describe the notion of $N_{\mathrm{PF}}$ and their fundamental properties. In Section 4, we present some novel contractive conditions based on $N_{\mathrm{PF}}$. By using these contractive conditions, we instigate some fixed point theorems for a picture fuzzy $n$-Banach space $\left(B_{\mathrm{PF}}\right)$. Finally, the conclusion of this manuscript is discussed in Section 5.

\section{Preliminaries}

The purpose of this section is to review some existing notions, like $N_{\text {IF }}$ and their related properties. Throughout this section, the symbols $R_{\mathrm{Rn}}^{+}, R_{R n}, N_{\mathrm{Nn}}, X_{\mathrm{UNI}}, M_{m}, N_{n}$, *ct, and ${ }_{c \text { ctc }}$ represent the positive real numbers, real numbers, natural numbers, universal set, supporting grade, supporting against, continuous $t$-norm, and continuous $t$-conorm, respectively.

Definition 1. [9]. A $N_{\mathrm{IF}}$ is stated by $\left(X_{\mathrm{UNI}}, M_{m}, A_{a}, N_{n}\right.$, $*_{\text {ct }}$, $\left.{ }^{c t c}\right)$, where $M_{m}, A_{a}, N_{n}$ is defined on $\left(x_{1}, x_{2}, \cdots, x_{k}, p\right)$ $\in X_{\mathrm{UNI}}^{k} \times(0, \infty)$, where the following conditions hold:

(i) $M_{m}\left(x_{1}, x_{2}, \cdots, x_{k}, p\right)+A_{a}\left(x_{1}, x_{2}, \cdots, x_{k}, p\right)+N_{n}\left(x_{1}\right.$, $\left.x_{2}, \cdots, x_{k}, p\right) \leq 1$

(ii) $M_{m}\left(x_{1}, x_{2}, \cdots, x_{k}, p\right)>0$

(iii) $M_{m}\left(\mathfrak{x}_{1}, \mathfrak{x}_{2}, \cdots, \mathfrak{x}_{k}, p\right)=1$ iff $\mathfrak{x}_{1}, \mathfrak{x}_{2}, \cdots, \mathfrak{x}_{k}$ are linearly dependent

(iv) $M_{m}\left(x_{1}, x_{2}, \cdots, x_{k}, p\right)$ is invariant under any permutation of $x_{1}, x_{2}, \cdots, x_{k}$

(v) $M_{m}\left(x_{1}, x_{2}, \cdots, \alpha x_{k}, p\right)=M_{m}\left(x_{1}, x_{2}, \cdots, x_{k}, p /|\alpha|\right)$ if $\alpha \neq 0 \in \mathbb{R}_{\mathrm{Rn}}$

(vi) $M_{m}\left(x_{1}, x_{2}, \cdots, x_{k}+x_{k}^{\prime}, p+q\right) \geq \min \left(M_{m}\left(x_{1}, x_{2}, \cdots\right.\right.$, $\left.\left.x_{k}, p\right), M_{m}\left(x_{1}, x_{2}, \cdots, x_{k}^{\prime}, q\right)\right)$

(vii) $M_{m}\left(x_{1}, x_{2}, \cdots, x_{k}+x_{k},.\right)$ is a nondecreasing function of $\mathbb{R}_{\mathrm{Rn}}^{++}$and $\log _{p \rightarrow \infty} M_{m}\left(x_{1}, x_{2}, \cdots, x_{k}+\right.$ $\left.x_{k}, p\right)=1$

(viii) $N_{n}\left(x_{1}, x_{2}, \cdots, x_{k}, p\right)<1$

(ix) $N_{n}\left(x_{1}, x_{2}, \cdots, x_{k}, p\right)=0$ iff $x_{1}, x_{2}, \cdots, x_{k}$ are linearly dependent (x) $N_{n}\left(x_{1}, x_{2}, \cdots, x_{k}, p\right)$ is invariant under any permutation of $x_{1}, x_{2}, \cdots, x_{k}$

(xi) $N_{n}\left(x_{1}, x_{2}, \cdots, \alpha x_{k}, p\right)=N_{n}\left(x_{1}, x_{2}, \cdots, x_{k}, p /|\alpha|\right)$ if $\alpha \neq 0 \in \mathbb{R}_{\mathrm{Rn}}$

(xii) $N_{n}\left(x_{1}, x_{2}, \cdots, x_{k}+x_{k}^{\prime}, p+q\right) \geq \max \left(N_{n}\left(x_{1}, x_{2}, \cdots\right.\right.$, $\left.\left.x_{k}, p\right), N_{n}\left(x_{1}, x_{2}, \cdots, x_{k}^{\prime}, q\right)\right)$

(xiii) $N_{n}\left(x_{1}, x_{2}, \cdots, x_{k}+x_{k}\right.$, .) is a nonincreasing function of $\mathbb{R}_{\mathrm{Rn}}^{+}$and $\log _{p \rightarrow \infty} N_{n}\left(x_{1}, x_{2}, \cdots, x_{k}+x_{k}, p\right)=0$

(xiv) Further, $M_{n}\left(x_{1}, x_{2}, \cdots, x_{k}, p\right)>0$ and $N_{n}\left(x_{1}, x_{2}, \cdots\right.$, $\left.\mathfrak{x}_{k}, p\right)<1$ imply $\mathfrak{x}=0, \forall p>0$

(xv) For $p \neq 0, M_{n}\left(x_{1}, x_{2}, \cdots, x_{k},.\right)$ and $N_{n}\left(x_{1}, x_{2}, \cdots, x_{k},.\right)$ are continuous functions of $\mathbb{R}_{\mathrm{Rn}}^{+}$and are strictly increasing and strictly decreasing, respectively, on the subset $\left\{p: 0<M_{m}\left(x_{1}, x_{2}, \cdots, x_{k}, p\right), N_{n}\left(x_{1}, x_{2}\right.\right.$, $\left.\left.\cdots, x_{k}, p\right)<1\right\}$ of $\mathbb{R}_{\mathrm{Rn}}^{+}$

Moreover, we explain some important theories based on convergent and Cauchy convergent sequences.

Definition 2. [9]. Consider $N_{\mathrm{IF}}\left(X_{\mathrm{UNI}}, M_{m}, N_{n}, *_{\mathrm{ct}},{ }^{\circ} \mathrm{ctc}\right)$; then, the sequence $x=\left\{x_{\mathfrak{f}}\right\}$ in $X_{\mathrm{UNI}}$ is convergent to $g \in$ $X_{\text {UNI }}$ based on the intuitionistic fuzzy $n$-norm $\left(M_{m}, N_{n}\right)^{k}$ if for every $\epsilon, p>0$ and $\ddot{y}_{1}, \ddot{y}_{2}, \cdots, \ddot{y}_{k-1} \in X_{\mathrm{UNI}}$, there exists $\tilde{\omega}_{0}$ $\in \mathbb{N}_{\mathrm{Nn}}$ such that

$$
\begin{aligned}
& M_{n}\left(\ddot{y}_{1}, \ddot{y}_{2}, \cdots, \ddot{y}_{k-1}, x_{\tilde{\omega}}-\boldsymbol{g}, p\right)>1-\epsilon, \\
& N_{n}\left(\ddot{y}_{1}, \ddot{y}_{2}, \cdots, \ddot{y}_{k-1}, x_{\tilde{\omega}}-\boldsymbol{g}, p\right)<\epsilon,
\end{aligned}
$$

for all $\tilde{\omega} \geq \tilde{\omega}_{0}$ and it is represented by $\left(M_{m}, N_{n}\right)^{k}-\lim x_{\tilde{\omega}}=\boldsymbol{g}$.

Definition 3. [9]. Let $N_{\mathrm{IF}}\left(X_{\mathrm{UNI}}, M_{m}, N_{n}, *_{\mathrm{ct}}\right.$, $\left.{ }_{\mathrm{c}} \mathrm{ctc}\right)$; then, the sequence $x=\left\{x_{\mathfrak{f}}\right\}$ in $X_{\mathrm{UNI}}$ is Cauchy convergent based on the intuitionistic fuzzy $n$-norm $\left(M_{m}, N_{n}\right)^{k}$ if for every $\epsilon$, $p>0$ and $\ddot{y}_{1}, \ddot{y}_{2}, \cdots, y_{k-1} \in X_{\mathrm{UNI}}$, there exists $\tilde{\omega}_{0} \in \mathbb{N}_{\mathrm{Nn}}$ such that

$$
\begin{aligned}
& M_{n}\left(\ddot{y}_{1}, \ddot{y}_{2}, \cdots, \ddot{y}_{k-1}, x_{\tilde{\omega}}-x_{\gamma}, p\right)>1-\epsilon, \\
& N_{n}\left(\ddot{y}_{1}, \ddot{y}_{2}, \cdots, \ddot{y}_{k-1}, x_{\tilde{\omega}}-x_{\gamma}, p\right)<\epsilon,
\end{aligned}
$$

for all $\tilde{\omega}, " \Upsilon \geq \tilde{\omega}_{0}$ and it is represented by $\left(M_{m}, N_{n}\right)^{k}-$ $\lim x_{\tilde{\omega}}=x_{Y}$.

\section{Picture Fuzzy $n$-Normed Linear Space}

The purpose of this section is to explore some new approaches like $N_{\mathrm{PF}}$ and their related properties, which are extensively efficient for the proof of our main work in the next section. Throughout this section, the symbols $X_{\mathrm{UNI}}$, $M_{m}, A_{a}, N_{n}$, ${ }_{c t}$, and ${ }_{c t c}$ represented the universal set, supporting grade, abstinence grade, supporting against, continuous $t$-norm, and continuous $t$-conorm, respectively. 
Definition 4. A $N_{\mathrm{PF}}$ is stated as $\left(X_{\mathrm{UNI}}, M_{m}, A_{a}, N_{n}\right.$, $*_{\mathrm{ct}}$, $\left.{ }_{\mathrm{ctc}}\right)$, where $M_{m}, A_{a}, N_{n}$ is defined on $\left(x_{1}, x_{2}, \cdots, x_{k}, p\right) \in X_{\mathrm{UNI}}^{k} \times$ $(0, \infty)$, where the following conditions hold:

(i) $M_{m}\left(x_{1}, x_{2}, \cdots, x_{k}, p\right)+A_{a}\left(x_{1}, x_{2}, \cdots, x_{k}, p\right)+N_{n}\left(x_{1}\right.$ $\left., x_{2}, \cdots, x_{k}, p\right) \leq 1$

(ii) $M_{m}\left(\mathfrak{x}_{1}, \mathfrak{x}_{2}, \cdots, \mathfrak{x}_{k}, p\right)>0$

(iii) $M_{m}\left(x_{1}, x_{2}, \cdots, x_{k}, p\right)=1$ iff $x_{1}, x_{2}, \cdots, x_{k}$ are linearly dependent

(iv) $M_{m}\left(x_{1}, x_{2}, \cdots, x_{k}, p\right)$ is invariant under any permutation of $x_{1}, x_{2}, \cdots, x_{k}$

(v) $M_{m}\left(x_{1}, x_{2}, \cdots, \alpha x_{k}, p\right)=M_{m}\left(x_{1}, x_{2}, \cdots, x_{k}, p /|\alpha|\right)$ if $\alpha \neq 0 \in \mathbb{R}_{\mathrm{Rn}}$

(vi) $M_{m}\left(x_{1}, x_{2}, \cdots, x_{k}+x_{k}^{\prime}, p+q\right) \geq \min \left(M_{m}\left(x_{1}, x_{2}\right.\right.$, $\left.\left.\cdots, x_{k}, p\right), M_{m}\left(x_{1}, x_{2}, \cdots, x_{k}^{\prime}, q\right)\right)$

(vii) $M_{m}\left(x_{1}, x_{2}, \cdots, x_{k}+x_{k}\right.$.) is a nondecreasing function of $\mathbb{R}_{\mathrm{Rn}}^{+}$and $\log _{p \rightarrow \infty} M_{m}\left(x_{1}, x_{2}, \cdots, x_{k}+x_{k}\right.$, $p)=1$

(viii) $A_{a}\left(x_{1}, x_{2}, \cdots, x_{k}, p\right)<1$

(ix) $A_{a}\left(x_{1}, x_{2}, \cdots, x_{k}, p\right)=0$ iff $x_{1}, x_{2}, \cdots, x_{k}$ are linearly dependent

(x) $A_{a}\left(x_{1}, x_{2}, \cdots, x_{k}, p\right)$ is invariant under any permutation of $x_{1}, x_{2}, \cdots, x_{k}$

(xi) $A_{a}\left(x_{1}, x_{2}, \cdots, \alpha x_{k}, p\right)=A_{a}\left(x_{1}, x_{2}, \cdots, x_{k}, p /|\alpha|\right)$ if $\alpha \neq 0 \in \mathbb{R}_{\mathrm{Rn}}$

(xii) $A_{a}\left(x_{1}, x_{2}, \cdots, x_{k}+x_{k}^{\prime}, p+q\right) \geq \max \left(A_{a}\left(x_{1}, x_{2}, \cdots\right.\right.$, $\left.\left.x_{k}, p\right), A_{a}\left(x_{1}, x_{2}, \cdots, x_{k}^{\prime}, q\right)\right)$

(xiii) $A_{a}\left(x_{1}, x_{2}, \cdots, x_{k}+x_{k}\right.$, .) is a nonincreasing function of $\mathbb{R}_{\mathrm{Rn}}^{+}$and $\log _{p \rightarrow \infty} A_{a}\left(x_{1}, x_{2}, \cdots, x_{k}+x_{k}, p\right)=0$

(xiv) $N_{n}\left(x_{1}, x_{2}, \cdots, x_{k}, p\right)<1$

(xv) $N_{n}\left(x_{1}, x_{2}, \cdots, x_{k}, p\right)=0$ iff $x_{1}, x_{2}, \cdots, x_{k}$ are linearly dependent

(xvi) $N_{n}\left(x_{1}, x_{2}, \cdots, x_{k}, p\right)$ is invariant under any permutation of $x_{1}, x_{2}, \cdots, x_{k}$

(xvii) $N_{n}\left(x_{1}, x_{2}, \cdots, \alpha x_{k}, p\right)=N_{n}\left(x_{1}, x_{2}, \cdots, x_{k}, p /|\alpha|\right)$ if $\alpha \neq 0 \in \mathbb{R}_{\mathrm{Rn}}$

(xviii) $N_{n}\left(x_{1}, x_{2}, \cdots, x_{k}+x_{k}^{\prime}, p+q\right) \geq \max \left(N_{n}\left(x_{1}, x_{2}, \cdots\right.\right.$, $\left.\left.x_{k}, p\right), N_{n}\left(x_{1}, x_{2}, \cdots, x_{k}^{\prime}, q\right)\right)$

(xix) $\left(x_{1}, x_{2}, \cdots, x_{k}+x_{k},.\right)$ is a nonincreasing function of $\mathbb{R}_{\mathrm{Rn}}^{+}$and $\log _{p \rightarrow \infty} N_{n}\left(x_{1}, x_{2}, \cdots, x_{k}+x_{k}, p\right)=0$

(xx) Further, $M_{n}\left(x_{1}, x_{2}, \cdots, x_{k}, p\right)>0, A_{n}\left(x_{1}, x_{2}, \cdots, x_{k}\right.$, $p)<1$ and $N_{n}\left(x_{1}, x_{2}, \cdots, x_{k}, p\right)<1$; then, $x=0$, $\forall p>0$

(xxi) For $p \neq 0, M_{n}\left(x_{1}, x_{2}, \cdots, x_{k} ..\right), A_{n}\left(x_{1}, x_{2}, \cdots, x_{k}.\right)$, and $N_{n}\left(x_{1}, x_{2}, \cdots, x_{k}, \cdot\right)$ are continuous functions of $\mathbb{R}_{\mathrm{Rn}}^{+}$and also strictly increasing and strictly decreasing, respectively, on the subset $\{p: 0<$ $M_{m}\left(x_{1}, x_{2}, \cdots, x_{k}, p\right), A_{a}\left(x_{1}, x_{2}, \cdots, x_{k}, p\right), N_{n}\left(x_{1}\right.$, $\left.\left.x_{2}, \cdots, x_{k}, p\right)<1\right\}$ of $\mathbb{R}_{\mathrm{Rn}}^{+}$

Moreover, we explain some important theories based on convergent and Cauchy convergent sequences.

Definition 5. For a $N_{\mathrm{PF}}\left(X_{\mathrm{UNI}}, M_{m}, A_{a}, N_{n}, *_{\mathrm{ct}},{ }^{\circ} \mathrm{ctc}\right)$, the sequence $\mathcal{X}=\left\{x_{\mathfrak{f}}\right\}$ in $X_{\mathrm{UNI}}$ is convergent to $\boldsymbol{g} \in X_{\mathrm{UNI}}$ based on the picture fuzzy $n$-norm $\left(M_{m}, A_{a}, N_{n}\right)^{k}$ if for every $\epsilon, p>0$ and $\tilde{y}_{1}, \ddot{y}_{2}, \cdots, y_{k-1} \in X_{\mathrm{UNI}}$, there exists $\tilde{\omega}_{0} \in \mathbb{N}_{\mathrm{Nn}}$ such that

$$
\begin{aligned}
& M_{n}\left(\ddot{y}_{1}, \ddot{y}_{2}, \cdots, \ddot{y}_{k-1}, x_{\tilde{\omega}}-\boldsymbol{g}, p\right)>1-\epsilon, \\
& A_{n}\left(\ddot{y}_{1}, \ddot{y}_{2}, \cdots, \ddot{y}_{k-1}, x_{\tilde{\omega}}-\boldsymbol{g}, p\right)<\epsilon, \\
& N_{n}\left(\ddot{y}_{1}, \ddot{y}_{2}, \cdots, \ddot{y}_{k-1}, x_{\tilde{\omega}}-\boldsymbol{g}, p\right)<\epsilon,
\end{aligned}
$$

for all $\tilde{\omega} \geq \tilde{\omega}_{0}$ and it is represented by $\left(M_{m}, A_{a}, N_{n}\right)^{k}-$ $\lim x_{\tilde{\omega}}=g$.

Definition 6. For a $N_{\mathrm{PF}}\left(X_{\mathrm{UNI}}, M_{m}, A_{a}, N_{n}, *_{\mathrm{ct}},{ }_{\mathrm{ctc}}\right)$, the sequence $\mathcal{X}=\left\{x_{\mathrm{f}}\right\}$ in $X_{\mathrm{UNI}}$ is Cauchy convergent based on the picture fuzzy $n$-norm $\left(M_{m}, A_{a}, N_{n}\right)^{k}$ if for every $\epsilon, p>$ 0 and $\ddot{y}_{1}, \ddot{y}_{2}, \cdots, \ddot{y}_{k-1} \in X_{\mathrm{UNI}}$, there exists $\tilde{\omega}_{0} \in \mathbb{N}_{\mathrm{Nn}}$ such that

$$
\begin{aligned}
& M_{n}\left(\ddot{y}_{1}, \ddot{y}_{2}, \cdots, \ddot{y}_{k-1}, x_{\tilde{\omega}}-x_{\gamma}, p\right)>1-\epsilon, \\
& A_{n}\left(\ddot{y}_{1}, \ddot{y}_{2}, \cdots, \ddot{y}_{k-1}, x_{\tilde{\omega}}-x_{\gamma}, p\right)<\epsilon, \\
& N_{n}\left(\ddot{y}_{1}, \ddot{y}_{2}, \cdots, \ddot{y}_{k-1}, x_{\tilde{\omega}}-x_{\gamma}, p\right)<\epsilon,
\end{aligned}
$$

for all $\tilde{\omega}, " \Upsilon \geq \tilde{\omega}_{0}$ and it is represented by $\left(M_{m}, A_{a}, N_{n}\right)^{k}-$ $\lim x_{\tilde{\omega}}=x^{\prime} \gamma$.

Remark 7. The following assumptions are important for our main results.

(1) Suppose $S_{m-1}$ is the set of functions $\Psi_{m-1}:[0,+\infty)$ $\longrightarrow[0,+\infty)$ such that

(i) $\Psi_{m-1}$ is continuous and nondecreasing

(ii) $\Psi_{m-1}(p)=0 \Leftrightarrow p=0$

(2) Suppose $S_{a-2}, S_{n-3}$ is the set of functions $\Psi_{a-2}$, $\Psi_{n-3}:[0,+\infty) \longrightarrow[0,+\infty)$ such that

(i) $\Psi_{a-2}, \Psi_{n-3}$ is continuous and nonincreasing

(ii) $\Psi_{a-2}(p), \Psi_{n-3}(p)=0 \Leftrightarrow p=0$

(3) Suppose $\bar{T}_{m-1}$ is the set of functions $\Theta_{1}:[0,+\infty)$ $\longrightarrow[0,+\infty)$ such that

(i) $\Theta_{1}$ is continuous and strictly increasing

(ii) $\Theta_{1}(p)=0 \Leftrightarrow p=0$ 
(4) Suppose $\bar{T}_{a-2}, \bar{T}_{n-3}$ is the set of functions with $\Theta_{2}$, $\Theta_{3}:[0,+\infty) \longrightarrow[0,+\infty)$ such that

(i) $\Theta_{2}, \Theta_{3}$ is continuous and strictly decreasing

(ii) $\Theta_{2}(p), \Theta_{3}(p)=0 \Leftrightarrow p=0$

\section{Contractive Mappings Based on the Picture Fuzzy $n$-Banach Space}

Based on the definitions introduced in Section 3, we describe some contractive mappings using the $B_{\mathrm{PF}}$ named as picture fuzzy $n$-normed contractive mapping $\left(N_{\mathrm{CM}}\right)$ and verify it with the help of numerical examples.
Definition 8. For a $N_{\mathrm{PF}}\left(X_{\mathrm{UNI}}, M_{m}, A_{a}, N_{n}, *_{\mathrm{ct}}\right.$, $\left.{ }_{\mathrm{c}} \mathrm{ct}\right)$, the mapping $T: X_{\mathrm{UNI}} \longrightarrow X_{\mathrm{UNI}}$ is called $N_{\mathrm{CM}}$, if

$$
\left.\begin{array}{l}
M_{n}\left(x_{1}, x_{2}, \cdots, x_{k-1}, x-y ̈, p\right) \leq M_{n}\left(x_{1}, x_{2}, \cdots, x_{k-1}, T(x)-T(\ddot{y}), p\right) \\
A_{n}\left(x_{1}, x_{2}, \cdots, x_{k-1}, x-y, p\right) \geq A_{n}\left(x_{1}, x_{2}, \cdots, x_{k-1}, T(x)-T(y), p\right) \\
N_{n}\left(x_{1}, x_{2}, \cdots, x_{k-1}, x-y, p\right) \geq N_{n}\left(x_{1}, x_{2}, \cdots, x_{k-1}, T(x)-T(y), p\right)
\end{array}\right\},
$$

for all $x_{1}, x_{2}, \cdots, x_{k-1} \in X_{\mathrm{UNI}}, x, y \in X_{\mathrm{UNI}}, p>0$.

Further, based on equation (5) and using Remark 7, we explore the following results, which are very helpful for future work.

Theorem 9. For a $N_{P F}\left(X_{U N I}, M_{m}, A_{a}, N_{n}\right.$, ${ }_{c t}$, o $\left.c t c\right)$, we define $N_{C M}, T: X_{U N I} \longrightarrow X_{U N I}$ such that

$$
\left.\begin{array}{l}
M_{n}\left(x_{1}, x_{2}, \cdots, x_{k-1}, x-y, p\right) \geq \alpha \Longrightarrow M_{n}\left(x_{1}, x_{2}, \cdots, x_{k-1}, T(x)-T(y), p-\Psi_{m-1}(p)\right) \geq \alpha \\
A_{n}\left(x_{1}, x_{2}, \cdots, x_{k-1}, x-y, p\right)<1-\alpha \Longrightarrow A_{n}\left(x_{1}, x_{2}, \cdots, x_{k-1}, T(x)-T(\ddot{y}), p-\Psi_{a-2}(p)\right)<1-\alpha \\
N_{n}\left(x_{1}, x_{2}, \cdots, x_{k-1}, x-y, p\right)<1-\alpha \Longrightarrow N_{n}\left(x_{1}, x_{2}, \cdots, x_{k-1}, T(x)-T\left(y^{\prime \prime}\right), p-\Psi_{n-3}(p)\right)<1-\alpha
\end{array}\right\},
$$

where $\Psi_{m-1} \in S_{m-1}, \Psi_{a-2} \in S_{a-2}$, and $\Psi_{n-3} \in S_{n-3}$, for all $x_{1}$, $x_{2}, \cdots, x_{k-1} \in X_{U N I}, x, y \in X_{U N I}, p>0$ with $\alpha \in(0,1]$. Then, $T$ possesses a unique fixed point in $X_{U N I}$.

Proof. Let $x_{0} \in X_{\mathrm{UNI}}$ with $x_{k+1}=T\left(x_{k}\right) \forall k \in \mathbb{N}_{\mathrm{Nn}}$. By using Remark 7 and inequality (6), we get

$$
\begin{aligned}
& M_{n}\left(x_{1}, x_{2}, \cdots, x_{k-1}, x-\ddot{y}, p\right) \\
& \leq M_{n}\left(\mathfrak{x}_{1}, \mathfrak{x}_{2}, \cdots, \mathfrak{x}_{k-1}, T(\mathfrak{x})-T(\ddot{y}), p-\Psi_{m-1}(p)\right), \\
& A_{n}\left(x_{1}, x_{2}, \cdots, x_{k-1}, \mathfrak{x}-\ddot{y}, p\right) \\
& \geq A_{n}\left(x_{1}, x_{2}, \cdots, x_{k-1}, T(x)-T(\ddot{y}), p-\Psi_{a-2}(p)\right), \\
& N_{n}\left(\mathfrak{x}_{1}, \mathfrak{x}_{2}, \cdots, \mathfrak{x}_{k-1}, \mathfrak{x}-\ddot{y}, p\right) \\
& \geq N_{n}\left(x_{1}, x_{2}, \cdots, x_{k-1}, T(x)-T(y), p-\Psi_{n-3}(p)\right) .
\end{aligned}
$$

Further, we write the above equations as

$$
\begin{aligned}
& M_{n}\left(x_{1}, x_{2}, \cdots, x_{k-1}, x_{k+1}-x_{k}, p\right) \\
& \quad \leq M_{n}\left(x_{2}, x_{3}, \cdots, x_{k}, x_{k+2}-x_{k+1}, p-\Psi_{m-1}(p)\right) \\
& \quad \leq M_{n}\left(x_{2}, x_{3}, \cdots, x_{k}, x_{k+2}-x_{k+1}, p\right), \\
& \quad A_{n}\left(x_{1}, x_{2}, \cdots, x_{k-1}, x_{k+1}-x_{k}, p\right) \\
& \quad \geq A_{n}\left(x_{2}, x_{3}, \cdots, x_{k}, x_{k+2}-x_{k+1}, p-\Psi_{a-2}(p)\right) \\
& \quad \geq A_{n}\left(x_{2}, x_{3}, \cdots, x_{k}, x_{k+2}-x_{k+1}, p\right), \\
& N_{n}\left(x_{1}, x_{2}, \cdots, x_{k-1}, x_{k+1}-x_{k}, p\right) \\
& \quad \geq N_{n}\left(x_{2}, x_{3}, \cdots, x_{k}, x_{k+2}-x_{k+1}, p-\Psi_{n-3}(p)\right) \\
& \quad \leq N_{n}\left(x_{2}, x_{3}, \cdots, x_{k}, x_{k+2}-x_{k+1}, p\right) .
\end{aligned}
$$

It is clear from the above analysis that $\left\{M_{n}\left(x_{1}, x_{2}\right.\right.$, $\left.\left.\cdots, x_{k-1}, x_{k+1}-x_{k}, p\right)\right\}$ is a bounded nondecreasing sequence while $\left\{A_{n}\left(\mathfrak{x}_{1}, \mathfrak{x}_{2}, \cdots, \mathfrak{x}_{k-1}, \mathfrak{x}_{k+1}-\mathfrak{x}_{k}, p\right)\right\}$ and $\left\{N_{n}\left(\mathfrak{x}_{1}, \mathfrak{x}_{2}, \cdots\right.\right.$, $\left.\left.\mathfrak{x}_{k-1}, \mathfrak{x}_{k+1}-\mathfrak{x}_{k}, p\right)\right\}$ are bounded nonincreasing sequences. Then, the limit of these equations exists. Hence,

$$
\begin{aligned}
& M_{n}\left(x_{2}, x_{3}, \cdots, x_{k-1}, x_{1}-x_{0}, p+\Psi_{m-1}(p)\right) \\
& \quad \leq M_{n}\left(x_{2}, x_{3}, \cdots, x_{k}, x_{2}-x_{1}, p+\Psi_{m-1}(p)-\Psi_{m-1}\left(p+\Psi_{m-1}(p)\right)\right) \\
& \quad \leq M_{n}\left(x_{2}, x_{3}, \cdots, x_{k}, x_{2}-x_{1}, p\right), \\
& \quad A_{n}\left(x_{2}, x_{3}, \cdots, x_{k-1}, x_{1}-x_{0}, p+\Psi_{a-1}(p)\right) \\
& \quad \geq A_{n}\left(x_{2}, x_{3}, \cdots, x_{k}, x_{2}-x_{1}, p\right), \\
& N_{n}\left(x_{2}, x_{3}, \cdots, x_{k-1}, x_{1}-x_{0}, p+\Psi_{n-1}(p)\right) \\
& \quad \geq N_{n}\left(x_{2}, x_{3}, \cdots, x_{k}, x_{2}-x_{1}, p\right) .
\end{aligned}
$$

By using the induction on $k$, we have

$$
\begin{aligned}
& M_{n}\left(\mathfrak{x}_{2}, \mathfrak{x}_{3}, \cdots, \mathfrak{x}_{k-1}, \mathfrak{x}_{1}-\mathfrak{x}_{0}, p+\Psi_{m-1}(p)\right) \\
& \quad \leq M_{n}\left(\mathfrak{x}_{1}, \mathfrak{x}_{2}, \cdots, \mathfrak{x}_{k-1}, \mathfrak{x}_{k+1}-\mathfrak{x}_{k}, p\right), \\
& \quad A_{n}\left(\mathfrak{x}_{2}, \mathfrak{x}_{3}, \cdots, \mathfrak{x}_{k-1}, \mathfrak{x}_{1}-\mathfrak{x}_{0}, p+\Psi_{a-1}(p)\right) \\
& \quad \geq A_{n}\left(\mathfrak{x}_{1}, \mathfrak{x}_{2}, \cdots, \mathfrak{x}_{k-1}, \mathfrak{x}_{k+1}-\mathfrak{x}_{k}, p\right), \\
& N_{n}\left(\mathfrak{x}_{2}, \mathfrak{x}_{3}, \cdots, \mathfrak{x}_{k-1}, \mathfrak{x}_{1}-\mathfrak{x}_{0}, p+\Psi_{n-1}(p)\right) \\
& \quad \geq N_{n}\left(\mathfrak{x}_{1}, \mathfrak{x}_{2}, \cdots, \mathfrak{x}_{k-1}, \mathfrak{x}_{k+1}-\mathfrak{x}_{k}, p\right) .
\end{aligned}
$$


As $k \longrightarrow \infty$, we have

$$
\begin{aligned}
& \lim _{k \rightarrow \infty} M_{n}\left(\mathfrak{x}_{1}, \mathfrak{x}_{2}, \cdots, \mathfrak{x}_{k-1}, \mathfrak{x}_{k+1}-\mathfrak{x}_{k}, p\right)=1, \\
& \lim _{k \rightarrow \infty} A_{n}\left(\mathfrak{x}_{1}, \mathfrak{x}_{2}, \cdots, \mathfrak{x}_{k-1}, \mathfrak{x}_{k+1}-\mathfrak{x}_{k}, p\right)=0, \\
& \lim _{k \rightarrow \infty} N_{n}\left(\mathfrak{x}_{1}, \mathfrak{x}_{2}, \cdots, \mathfrak{x}_{k-1}, \mathfrak{x}_{k+1}-\mathfrak{x}_{k}, p\right)=0 .
\end{aligned}
$$

Supposing $p, \epsilon>0$, we have

$$
\begin{array}{r}
M_{n}\left(\mathfrak{x}_{1}, \mathfrak{x}_{2}, \cdots, \mathfrak{x}_{k-1}, \mathfrak{x}_{K+1}-\mathfrak{x}_{K}, \frac{p}{2}\right) \geq 1-\epsilon, \\
M_{n}\left(\mathfrak{x}_{1}, \mathfrak{x}_{2}, \cdots, \mathfrak{x}_{k-1}, \mathfrak{x}_{K+1}-\mathfrak{x}_{K}, \Psi_{m-1}\left(\frac{p}{2}\right)\right) \geq 1-\epsilon .
\end{array}
$$

Similarly, from abstinence and falsity grades, we have

$$
\begin{aligned}
& A_{n}\left(x_{1}, x_{2}, \cdots, x_{k-1}, x_{K+1}-x_{K}, \Psi_{a-2}\left(\frac{p}{2}\right)\right)<\epsilon, \\
& N_{n}\left(x_{1}, x_{2}, \cdots, x_{k-1}, x_{K+1}-x_{K}, \Psi_{n-3}\left(\frac{p}{2}\right)\right)<\epsilon .
\end{aligned}
$$

By using the above analysis, we write, if $M_{n}\left(x_{1}, x_{2}, \cdots\right.$, $\left.\mathfrak{x}_{k-1}, \mathfrak{x}-\mathfrak{x}_{K},(p / 2)\right) \geq 1-\epsilon$ and $A_{n}\left(\mathfrak{x}_{1}, \mathfrak{x}_{2}, \cdots, \mathfrak{x}_{k-1}, \mathfrak{x}-\mathfrak{x}_{K}, p /\right.$ $2), N_{n}\left(x_{1}, x_{2}, \cdots, x_{k-1}, x-x_{K}, p / 2\right)<\epsilon$, then

$$
\begin{aligned}
& M_{n}\left(x_{1}, x_{2}, \cdots, x_{k-1}, T(x)-x_{K}, \frac{p}{2}\right) \\
& \quad \geq \min \left(M_{n}\left(x_{1}, x_{2}, \cdots, x_{k-1}, T(x)-T\left(x_{K}\right), \frac{p}{2}-\Psi_{m-1}\left(\frac{p}{2}\right)\right)\right), \\
& M_{n}\left(x_{1}, x_{2}, \cdots, x_{k-1}, T(x)-x_{K}, \Psi_{m-1}\left(\frac{p}{2}\right)\right) \\
& \quad \geq \min \left(M_{n}\left(x_{1}, x_{2}, \cdots, x_{k-1}, x-x_{K}, \frac{p}{2}\right)\right), \\
& M_{n}\left(x_{1}, x_{2}, \cdots, x_{k-1}, x_{K+1}-x_{K}, \Psi_{m-1}\left(\frac{p}{2}\right)\right) \geq 1-\epsilon .
\end{aligned}
$$

Similarly, solving the grades of abstinence and falsity, we have

$$
\begin{aligned}
& A_{n}\left(x_{1}, x_{2}, \cdots, x_{k-1}, \mathfrak{x}_{K+1}-\mathfrak{x}_{K}, \Psi_{a-2}\left(\frac{p}{2}\right)\right)<\epsilon, \\
& N_{n}\left(x_{1}, x_{2}, \cdots, x_{k-1}, x_{K+1}-\mathfrak{x}_{K}, \Psi_{n-3}\left(\frac{p}{2}\right)\right)<\epsilon .
\end{aligned}
$$

Therefore,

$$
M_{n}\left(x_{1}, x_{2}, \cdots, x_{k-1}, \mathfrak{x}_{k}-\mathfrak{x}_{K}, \frac{p}{2}\right) \geq 1-\epsilon
$$

Similarly, dealing with the grades of abstinence and falsity, we have

$$
\begin{aligned}
& A_{n}\left(x_{1}, x_{2}, \cdots, x_{k-1}, x_{k}-x_{K}, \frac{p}{2}\right)<\epsilon, \\
& N_{n}\left(x_{1}, x_{2}, \cdots, x_{k-1}, x_{k}-x_{K}, \frac{p}{2}\right)<\epsilon .
\end{aligned}
$$

Then, for all $k \geq \mathbb{N}_{\mathrm{Nn}}$,

$$
\begin{aligned}
& M_{n}\left(x_{1}, x_{2}, \cdots, x_{k-1}, x_{k}-x_{\Upsilon}, p\right) \\
& \geq \min \left\{M_{n}\left(x_{1}, x_{2}, \cdots, x_{k-1}, x_{k}-x_{K}, p\right), M_{n}\right. \\
& \left.\cdot\left(x_{1}, x_{2}, \cdots, x_{k-1}, x_{Y}-x_{K}, p\right)\right\} \geq 1-\epsilon, \\
& M_{n}\left(x_{1}, x_{2}, \cdots, x_{k-1}, \mathfrak{x}_{\Upsilon}-\mathfrak{x}_{K}, p\right) \geq 1-\epsilon .
\end{aligned}
$$

Also, we find

$$
\begin{aligned}
& A_{n}\left(x_{1}, x_{2}, \cdots, x_{k-1}, x_{k}-x_{\gamma}, p\right)<\epsilon, \\
& N_{n}\left(x_{1}, x_{2}, \cdots, x_{k-1}, x_{k}-x_{\gamma}, p\right)<\epsilon .
\end{aligned}
$$

Since $\epsilon$ is arbitrary and the sequence $\left\{x_{\mathfrak{f}}\right\}$ is Cauchy, hence they are convergent. Therefore, $\lim \left\{x_{\mathfrak{f}}\right\}=x$.

Suppose $p, \epsilon>0$; then, there exists $k_{0} \in \mathbb{N}_{\mathrm{Nn}}$ such that

$$
\begin{array}{r}
M_{n}\left(x_{1}, x_{2}, \cdots, x_{k-1}, x_{k}-x, \frac{p}{2}\right) \geq 1-\epsilon, \\
M_{n}\left(x_{1}, x_{2}, \cdots, x_{k-1}, x-x_{k}, \Psi_{m-1}\left(\frac{p}{2}\right)\right) \geq 1-\epsilon .
\end{array}
$$

Moreover, doing the same process to abstinence and falsity grades, we obtain

$$
\begin{aligned}
& A_{n}\left(x_{1}, x_{2}, \cdots, x_{k-1}, x_{k}-x, \Psi_{a-2}\left(\frac{p}{2}\right)\right)<\epsilon, \\
& N_{n}\left(x_{1}, x_{2}, \cdots, x_{k-1}, x_{k}-x, \Psi_{n-3}\left(\frac{p}{2}\right)\right)<\epsilon,
\end{aligned}
$$

for all $k \geq k_{0}$. Hence,

$$
\begin{aligned}
& M_{n}\left(x_{1}, x_{2}, \cdots, x_{k-1}, T(x)-x, p\right) \\
& \geq \min \left(M_{n}\left(x_{1}, x_{2}, \cdots, x_{k-1}, T(x)-x_{k+1}, p-\Psi_{m-1}(p)\right)\right) \text {, } \\
& M_{n}\left(x_{1}, x_{2}, \cdots, x_{k-1}, x_{k+1}-\mathfrak{x}, \Psi_{m-1}(p)\right) \\
& \geq \min \left(M_{n}\left(x_{1}, x_{2}, \cdots, x_{k-1}, \mathfrak{x}-x_{k}, p\right)\right), \\
& M_{n}\left(\mathfrak{x}_{1}, \mathfrak{x}_{2}, \cdots, x_{k-1}, \mathfrak{x}_{k+1}-\mathfrak{x}, \Psi_{m-1}(p)\right) \geq 1-\epsilon, \\
& A_{n}\left(\mathfrak{x}_{1}, \mathfrak{x}_{2}, \cdots, \mathfrak{x}_{k-1}, T(\mathfrak{x})-\mathfrak{x}, p\right)<\epsilon, \\
& N_{n}\left(x_{1}, x_{2}, \cdots, x_{k-1}, T(x)-x, p\right)<\epsilon,
\end{aligned}
$$

for all $k \geq k_{0}$. Therefore,

$$
\begin{aligned}
& M_{n}\left(x_{1}, x_{2}, \cdots, x_{k-1}, T(x)-x, p\right)=1, \\
& A_{n}\left(x_{1}, x_{2}, \cdots, x_{k-1}, T(x)-x, p\right)=0, \\
& N_{n}\left(x_{1}, x_{2}, \cdots, x_{k-1}, T(x)-x, p\right)=0,
\end{aligned}
$$

for all $p>0$. Hence, $T(x)=x$; that is, $T$ has a fixed point in $X_{\text {UNI }}$. Next, we prove its uniqueness. For this, we suppose $y$ is another fixed point of $T$ in $X_{\mathrm{UNI}}$; then, 


$$
\begin{aligned}
& M_{n}\left(x_{1}, x_{2}, \cdots, x_{k-1}, x-y, p+k \Psi_{m-1}(p)\right) \\
& \quad \geq \min \left(M_{n}\left(x_{1}, x_{2}, \cdots, x_{k-1}, T(x)-T(y), p\right)\right) \\
& \quad=M_{n}\left(x_{1}, x_{2}, \cdots, x_{k-1}, x-\ddot{y}, p\right), \\
& A_{n}\left(x_{1}, x_{2}, \cdots, x_{k-1}, x-y, y+k \Psi_{a-2}(p)\right) \\
& \quad=M_{n}\left(x_{1}, x_{2}, \cdots, x_{k-1}, x-\ddot{y}, p\right), \\
& N_{n}\left(x_{1}, x_{2}, \cdots, x_{k-1}, x-\ddot{y}, p+k \Psi_{n-3}(p)\right) \\
& \quad=M_{n}\left(x_{1}, x_{2}, \cdots, x_{k-1}, \mathfrak{y}-\ddot{y}, p\right),
\end{aligned}
$$

for all $k \in \mathbb{N}_{\mathrm{Nn}}$ and $k \longrightarrow \infty$; then,

$$
\begin{gathered}
M_{n}\left(x_{1}, x_{2}, \cdots, x_{k-1}, \mathfrak{x}-\ddot{y}, p\right)=1, \\
A_{n}\left(x_{1}, x_{2}, \cdots, x_{k-1}, x-\ddot{y}, p\right)=0, \\
N_{n}\left(x_{1}, x_{2}, \cdots, x_{k-1}, x-\ddot{y}, p\right)=0,
\end{gathered}
$$

for all $p>0$. Hence, $x=\ddot{y}$. Thus, $T$ has a unique fixed point in $X_{\mathrm{UNI}}$.

Example 10. For a Banach space $\left(X_{\mathrm{UNI}},\|\|.\right)$, we define a mapping $T: X_{\mathrm{UNI}} \longrightarrow X_{\mathrm{UNI}}$ such that for all $x, y \in X_{\mathrm{UNI}}$,

$$
\begin{aligned}
& \|T(x)-T(\ddot{y})\| \leq\|x-\ddot{y}\|-\Psi_{m-1}\|x-\ddot{y}\|,
\end{aligned}
$$

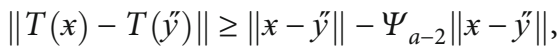

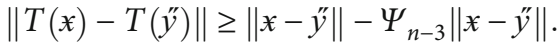

We know that $\Psi_{m-1} \in S_{m-1}, \Psi_{a-2} \in S_{a-2}$, and $\Psi_{n-3} \in S_{n-3}$. We consider that $\Psi_{m-1}(\beta p) \leq \beta \Psi_{m-1}(p), \quad \Psi_{a-2}(\beta p) \geq \beta$ $\Psi_{a-2}(p)$, and $\Psi_{n-3}(\beta p) \geq \beta \Psi_{n-3}(p)$, where $p>0$ and $\beta \in[0$, 1]. Now, we describe the picture fuzzy $n$-norm $\mathrm{M}_{n}, \mathrm{~A}_{n}$, and $\mathrm{N}_{n}$ :

$$
\begin{aligned}
& M_{n}\left(x_{1}, x_{2}, \cdots, x_{k-1}, x_{k}, p\right) \\
& \quad= \begin{cases}\frac{p}{\left\|x_{1}, x_{2}, \cdots, x_{k-1}, x_{k}\right\|}, & 0<p \leq\left\|x_{1}, x_{2}, \cdots, x_{k-1}, x_{k}\right\|, \\
1, & \left\|x_{1}, x_{2}, \cdots, x_{k-1}, x_{k}\right\|<p, \\
0, & p \leq 0,\end{cases}
\end{aligned}
$$$$
A_{n}\left(\mathfrak{x}_{1}, \mathfrak{x}_{2}, \cdots, \mathfrak{x}_{k-1}, \mathfrak{x}_{k}, p\right)
$$$$
= \begin{cases}1-\frac{p}{\left\|x_{1}, x_{2}, \cdots, x_{k-1}, x_{k}\right\|}, & 0<p \leq\left\|x_{1}, x_{2}, \cdots, x_{k-1}, x_{k}\right\|, \\ 0, & \left\|x_{1}, x_{2}, \cdots, x_{k-1}, x_{k}\right\|<p, \\ 1, & p \leq 0,\end{cases}
$$

$$
\begin{aligned}
& N_{n}\left(x_{1}, x_{2}, \cdots, x_{k-1}, x_{k}, p\right) \\
& \quad= \begin{cases}1-\frac{p}{\left\|x_{1}, x_{2}, \cdots, x_{k-1}, x_{k}\right\|}, & 0<p \leq\left\|x_{1}, x_{2}, \cdots, x_{k-1}, x_{k}\right\|, \\
0, & \left\|x_{1}, x_{2}, \cdots, x_{k-1}, x_{k}\right\|<p, \\
1, & p \leq 0 .\end{cases}
\end{aligned}
$$

We consider that

$$
\begin{aligned}
& M_{n}\left(x_{1}, x_{2}, \cdots, x_{k-1}, x-\ddot{y}, p\right) \geq \alpha, \\
& A_{n}\left(x_{1}, x_{2}, \cdots, x_{k-1}, x-\ddot{y}, p\right)<1-\alpha, \\
& N_{n}\left(x_{1}, x_{2}, \cdots, x_{k-1}, x-\ddot{y}, p\right)<1-\alpha .
\end{aligned}
$$

The first three parts are discussed for the truth grade. We have the following cases:

Case 1. Suppose $0<p \leq\left\|x_{1}, x_{2}, \cdots, x_{k-1}, x-y, p\right\|$; then,

$$
\begin{gathered}
M_{n}\left(x_{1}, x_{2}, \cdots, x_{k-1}, x-\ddot{y}, p\right)=\frac{p}{\left\|x_{1}, x_{2}, \cdots, x_{k-1}, x-y, p\right\|} \geq \alpha, \\
p \geq \alpha\left\|x_{1}, x_{2}, \cdots, x_{k-1}, x-y, p\right\| .
\end{gathered}
$$

Further, we write

$$
\begin{aligned}
& \alpha\left\|x_{1}, \mathfrak{x}_{2}, \cdots, \mathfrak{x}_{k-1}, T(x)-T(\ddot{y})\right\| \\
& \quad \leq \alpha\left\|x_{1}, \mathfrak{x}_{2}, \cdots, \mathfrak{x}_{k-1}, \mathfrak{x}-\ddot{y}\right\|-\alpha \Psi_{m-1}\left\|\mathfrak{x}_{1}, \mathfrak{x}_{2}, \cdots, \mathfrak{x}_{k-1}, \mathfrak{x}-\ddot{y}\right\| \\
& \quad \leq \alpha\left\|\mathfrak{x}_{1}, \mathfrak{x}_{2}, \cdots, \mathfrak{x}_{k-1}, \mathfrak{x}-\ddot{y}\right\|-\Psi_{m-1}\left(\alpha\left\|\mathfrak{x}_{1}, \mathfrak{x}_{2}, \cdots, \mathfrak{x}_{k-1}, \mathfrak{x}-\ddot{y}\right\|\right) \\
& \quad \leq p-\Psi_{m-1}(p) .
\end{aligned}
$$

Therefore, we get

$$
\begin{gathered}
M_{n}\left(x_{1}, x_{2}, \cdots, x_{k-1}, T(x)-T\left(y^{\prime \prime}\right), p-\Psi_{m-1}(p)\right) \\
=\frac{p-\Psi_{m-1}(p)}{\left\|x_{1}, x_{2}, \cdots, x_{k-1}, T(x)-T\left(y^{\prime \prime}\right)\right\|} \geq \alpha .
\end{gathered}
$$

Case 2. Suppose $\left\|x_{1}, x_{2}, \cdots, x_{k-1}, x_{k}\right\|<p$; then,

$$
\begin{aligned}
\left\|x_{1}, x_{2}, \cdots, x_{k-1}, T(x)-T(y)\right\| \leq & \left\|x_{1}, x_{2}, \cdots, x_{k-1}, x-y\right\| \\
& -\Psi_{m-1}\left\|x_{1}, x_{2}, \cdots, x_{k-1}, x-y\right\| \\
\leq & p-\Psi_{m-1}(p) .
\end{aligned}
$$

Therefore, we get

$$
M_{n}\left(x_{1}, x_{2}, \cdots, x_{k-1}, T(x)-T(y), p-\Psi_{m-1}(p)\right)=1 \geq \alpha .
$$

Case 3. Suppose $p \leq 0$ and $M_{n}\left(x_{1}, x_{2}, \cdots, x_{k-1}, x_{k}, p\right)=0$; then,

$$
M_{n}\left(x_{1}, x_{2}, \cdots, x_{k-1}, T(x)-T(\ddot{y}), p-\Psi_{m-1}(p)\right)=1 \geq \alpha .
$$

Similarly, we can prove these conditions for abstinence and falsity grades. Hence, the solution is completed. Further, we instigate more results based on $B_{\mathrm{PF}}$ to show the proficiency of the discussed results. 
Theorem 11. For a $B_{P F}\left(X_{U N I}, M_{m}, A_{a}, N_{n}, *_{c t},{ }_{c t c}\right)$, the grade of truth, abstinence, and falsity satisfies the conditions of Definition 4. Now, we define the decreasing mapping $\Gamma_{1}$ and increasing mappings $\Gamma_{2}$ and $\Gamma_{3}$, such that $\Gamma_{1}:(0,+\infty)$ $\longrightarrow[0,1)$ and $\Gamma_{2}:(0,+\infty) \longrightarrow[0,1), \Gamma_{3}:(0,+\infty) \longrightarrow[0$, 1) with $T: X_{U N I} \longrightarrow X_{U N I}$, such that

$$
\left.\begin{array}{l}
M_{n}\left(x_{1}, x_{2}, \cdots, x_{k-1}, x-\ddot{y}, p\right) \geq \alpha \Longrightarrow M_{n}\left(x_{1}, x_{2}, \cdots, x_{k-1}, T(x)-T\left(y^{\prime \prime}\right), \Theta_{1}^{-1}\left(\Gamma_{1}(P) \Theta_{1}(P)\right)\right) \geq \alpha \\
A_{n}\left(x_{1}, x_{2}, \cdots, x_{k-1}, x-\ddot{y}, p\right)<1-\alpha \Longrightarrow A_{n}\left(x_{1}, x_{2}, \cdots, x_{k-1}, T(x)-T(y), \Theta_{2}^{-1}\left(\Gamma_{2}(P) \Theta_{2}(P)\right)\right)<1-\alpha \\
N_{n}\left(x_{1}, x_{2}, \cdots, x_{k-1}, x-\ddot{y}, p\right)<1-\alpha \Longrightarrow N_{n}\left(x_{1}, x_{2}, \cdots, x_{k-1}, T(x)-T(\ddot{y}), \Theta_{3}^{-1}\left(\Gamma_{3}(P) \Theta_{3}(P)\right)\right)<1-\alpha
\end{array}\right\},
$$

where $\Theta_{1} \in \bar{T}_{m-1}, \Theta_{2} \in \bar{T}_{a-2}$, and $\Theta_{3} \in \bar{T}_{n-3}$, for all $x_{1}, x_{2}, \cdots$, $x_{k-1} \in X_{U N I}, x, y \in X_{U N I}, p>0$ with $\alpha \in(0,1]$. Then, $T$ has a unique fixed point in $\mathrm{X}_{U N I}$.

Proof. Let $x_{0} \in X_{\mathrm{UNI}}$ with $x_{k+1}=T\left(x_{k}\right) \forall k \in \mathbb{N}_{\mathrm{Nn}}$. By using Remark 7 and inequality (35), we get

$$
\begin{aligned}
& M_{n}\left(x_{1}, x_{2}, \cdots, x_{k-1}, \mathfrak{x}-\ddot{y}, p\right) \leq M_{n}\left(x_{1}, x_{2}, \cdots, x_{k-1}, T(x)\right. \\
& \left.-T\left(y^{\prime \prime}\right), \Theta_{1}^{-1}\left(\Gamma_{1}(P) \Theta_{1}(P)\right)\right) \text {, } \\
& A_{n}\left(x_{1}, x_{2}, \cdots, x_{k-1}, \mathfrak{x}-\ddot{y}, p\right) \geq A_{n}\left(x_{1}, x_{2}, \cdots, x_{k-1}, T(x)\right. \\
& \left.-T(\ddot{y}), \Theta_{2}^{-1}\left(\Gamma_{2}(P) \Theta_{2}(P)\right)\right) \text {, } \\
& N_{n}\left(\mathfrak{x}_{1}, \mathfrak{x}_{2}, \cdots, \mathfrak{x}_{k-1}, \mathfrak{x}-\ddot{y}, p\right) \geq N_{n}\left(\mathfrak{x}_{1}, \mathfrak{x}_{2}, \cdots, \mathfrak{x}_{k-1}, T(\mathfrak{x})\right. \\
& \left.-T\left(y^{\prime \prime}\right), \Theta_{3}^{-1}\left(\Gamma_{3}(P) \Theta_{3}(P)\right)\right) \text {. }
\end{aligned}
$$

Further, we write the above equations as

$$
\begin{aligned}
& M_{n}\left(x_{1}, x_{2}, \cdots, x_{k-1}, x_{k+1}-x_{k}, p\right) \\
& \quad \leq M_{n}\left(x_{2}, x_{3}, \cdots, x_{k}, x_{k+2}-x_{k+1}, \Theta_{1}^{-1}\left(\Gamma_{1}(P) \Theta_{1}(P)\right)\right) \\
& \quad \leq M_{n}\left(x_{2}, x_{3}, \cdots, x_{k}, x_{k+2}-x_{k+1}, p\right), \\
& \quad A_{n}\left(x_{1}, x_{2}, \cdots, x_{k-1}, x_{k+1}-x_{k}, p\right) \\
& \quad \geq A_{n}\left(x_{2}, x_{3}, \cdots, x_{k}, x_{k+2}-x_{k+1}, \Theta_{2}^{-1}\left(\Gamma_{2}(P) \Theta_{2}(P)\right)\right) \\
& \quad \geq A_{n}\left(x_{2}, x_{3}, \cdots, x_{k}, x_{k+2}-x_{k+1}, p\right), \\
& \quad N_{n}\left(x_{1}, x_{2}, \cdots, x_{k-1}, x_{k+1}-x_{k}, p\right) \\
& \quad \geq N_{n}\left(x_{2}, x_{3}, \cdots, x_{k}, x_{k+2}-x_{k+1}, \Theta_{3}^{-1}\left(\Gamma_{3}(P) \Theta_{3}(P)\right)\right) \\
& \quad \leq N_{n}\left(x_{2}, x_{3}, \cdots, x_{k}, x_{k+2}-x_{k+1}, p\right) .
\end{aligned}
$$

It is clear from the above analysis that $\left\{M_{n}\left(x_{1}, x_{2}, \cdots\right.\right.$, $\left.\left.x_{k-1}, x_{k+1}-x_{k}, p\right)\right\}$ is a bounded nondecreasing sequence and $\left\{A_{n}\left(x_{1}, x_{2}, \cdots, x_{k-1}, x_{k+1}-x_{k}, p\right)\right\}$ and $\left\{N_{n}\left(x_{1}, x_{2}, \cdots\right.\right.$, $\left.\left.x_{k-1}, x_{k+1}-x_{k}, p\right)\right\}$ are the bounded nonincreasing sequences. Then, the limit of these equations exists. We suppose that

$$
\begin{aligned}
& \lim _{k \rightarrow \infty} M_{n}\left(x_{1}, x_{2}, \cdots, x_{k-1}, x_{k+1}-x_{k}, p\right)<\beta_{1}<1, \\
& \lim _{k \rightarrow \infty} A_{n}\left(x_{1}, x_{2}, \cdots, x_{k-1}, x_{k+1}-x_{k}, p\right)>\beta_{2}>1, \\
& \lim _{k \rightarrow \infty} N_{n}\left(x_{1}, x_{2}, \cdots, x_{k-1}, x_{k+1}-x_{k}, p\right)>\beta_{3}>1 .
\end{aligned}
$$

Therefore, we have

$$
\begin{aligned}
& M_{n}\left(x_{2}, x_{3}, \cdots, x_{k}, x_{k+2}-x_{k+1}, q\right) \geq M_{n}\left(x_{1}, x_{2}, \cdots, x_{k-1}, x_{k+1}-x_{k}, q\right), \\
& A_{n}\left(x_{2}, x_{3}, \cdots, x_{k}, x_{k+2}-x_{k+1}, q\right) \leq A_{n}\left(x_{1}, x_{2}, \cdots, x_{k-1}, x_{k+1}-x_{k}, q\right), \\
& N_{n}\left(x_{2}, x_{3}, \cdots, x_{k}, x_{k+2}-x_{k+1}, q\right) \leq N_{n}\left(x_{1}, x_{2}, \cdots, x_{k-1}, x_{k+1}-x_{k}, q\right), \\
& 0<p \leq\left\|x_{2}, x_{3}, \cdots, x_{k}, x_{k+2}-x_{k+1}\right\|_{\beta_{1}} \leq\left\|x_{2}, x_{3}, \cdots, x_{k-1}, x_{k+1}-x_{k}\right\|_{\beta_{1}}, \\
& 0<p \leq\left\|x_{2}, x_{3}, \cdots, x_{k-1}, x_{k+1}-x_{k}\right\|_{\beta_{2}} \leq\left\|x_{2}, x_{3}, \cdots, x_{k}, x_{k+2}-x_{k+1}\right\|_{\beta_{2}}, \\
& 0<p \leq\left\|x_{2}, x_{3}, \cdots, x_{k-1}, x_{k+1}-x_{k}\right\|_{\beta_{3}} \leq\left\|x_{2}, x_{3}, \cdots, x_{k}, x_{k+2}-x_{k+1}\right\|_{\beta_{3}} .
\end{aligned}
$$

Then, the limit of these equations also exists. We have

$$
\begin{aligned}
& \lim _{k \rightarrow \infty}\left\|x_{1}, x_{2}, \cdots, x_{k-1}, x_{k+1}-x_{k}\right\|_{\beta_{1}}=b_{1}, \\
& \lim _{k \rightarrow \infty}\left\|x_{1}, x_{2}, \cdots, x_{k-1}, x_{k+1}-x_{k}\right\|_{\beta_{2}}=b_{2}, \\
& \lim _{k \rightarrow \infty}\left\|x_{1}, x_{2}, \cdots, x_{k-1}, x_{k+1}-x_{k}\right\|_{\beta_{3}}=b_{3} .
\end{aligned}
$$

If $M_{n}\left(x_{2}, x_{3}, \cdots, x_{k}, x_{k+2}-x_{k+1}, q\right) \geq \beta_{1}, A_{n}\left(x_{2}, x_{3}, \cdots, x_{k}\right.$, $\left.x_{k+2}-x_{k+1}, q\right)<\beta_{2}$, and $N_{n}\left(x_{2}, x_{3}, \cdots, x_{k}, x_{k+2}-x_{k+1}, q\right)<\beta_{3}$, then

$$
\begin{aligned}
& M_{n}\left(x_{2}, x_{3}, \cdots, x_{k}, x_{k+2}-x_{k+1}, \Theta_{1}^{-1}\left(\Gamma_{1}(P) \Theta_{1}(P)\right)\right) \\
& \quad \geq M_{n}\left(x_{1}, x_{2}, \cdots, x_{k-1}, x_{k+1}-x_{k}, q\right) \geq \beta_{1}, \\
& A_{n}\left(x_{2}, x_{3}, \cdots, x_{k}, x_{k+2}-x_{k+1}, \Theta_{2}^{-1}\left(\Gamma_{2}(P) \Theta_{2}(P)\right)\right) \\
& \quad \leq A_{n}\left(x_{1}, x_{2}, \cdots, x_{k-1}, x_{k+1}-x_{k}, q\right)<\beta_{2}, \\
& M_{n}\left(x_{2}, x_{3}, \cdots, x_{k}, x_{k+2}-x_{k+1}, \Theta_{3}^{-1}\left(\Gamma_{3}(P) \Theta_{3}(P)\right)\right) \\
& \quad \leq M_{n}\left(x_{1}, x_{2}, \cdots, x_{k-1}, x_{k+1}-\mathfrak{x}_{k}, q\right)<\beta_{3} .
\end{aligned}
$$

Therefore,

$$
\begin{aligned}
& \left\|x_{2}, x_{3}, \cdots, x_{k}, x_{k+2}-x_{k+1}\right\|_{\beta_{1}} \leq \Theta_{1}^{-1}\left(\Gamma_{1}(P) \Theta_{1}(P)\right), \\
& \Theta_{1}\left\|x_{2}, x_{3}, \cdots, x_{k}, x_{k+2}-x_{k+1}\right\|_{\beta_{1}} \leq\left(\Gamma_{1}(P) \Theta_{1}(P)\right) \\
& \quad \leq\left(\Gamma_{1}\left(\left\|x_{1}, x_{2}, \cdots, x_{k-1}, x_{k+1}-x_{k}\right\|_{\beta_{1}}\right) \Theta_{1}(P)\right) \\
& \quad \leq\left(\Gamma_{1}\left(b_{1}\right) \Theta_{1}(P)\right) .
\end{aligned}
$$


Similarly, we can find that

$$
\begin{aligned}
& \Theta_{2}\left\|x_{2}, x_{3}, \cdots, x_{k}, x_{k+2}-x_{k+1}\right\|_{\beta_{2}} \geq \Theta_{2}^{-1}\left(\Gamma_{2}(P) \Theta_{2}(P)\right) \\
& \geq\left(\Gamma_{2}\left(b_{2}\right) \Theta_{2}(P)\right), \\
& \Theta_{3}\left\|x_{2}, x_{3}, \cdots, x_{k}, x_{k+2}-x_{k+1}\right\|_{\beta_{3}} \geq \Theta_{3}^{-1}\left(\Gamma_{3}(P) \Theta_{3}(P)\right) \\
& \geq\left(\Gamma_{3}\left(b_{3}\right) \Theta_{3}(P)\right) .
\end{aligned}
$$

And it is clear that $p \longrightarrow\left\|x_{1}, x_{2}, \cdots, x_{k-1}, x_{k+1}-x_{k}\right\|_{\beta_{1}}$, $\left\|x_{1}, x_{2}, \cdots, x_{k-1}, x_{k+1}-x_{k}\right\|_{\beta_{2}},\left\|x_{1}, x_{2}, \cdots, x_{k-1}, x_{k+1}-x_{k}\right\|_{\beta_{3}}$; then,

$$
\begin{aligned}
& \Theta_{1}\left\|x_{2}, x_{3}, \cdots, x_{k}, x_{k+2}-x_{k+1}\right\|_{\beta_{1}} \\
& \quad \leq\left(\Gamma_{1}\left(b_{1}\right) \Theta_{1}\left(\left\|x_{1}, x_{2}, \cdots, x_{k-1}, x_{k+1}-x_{k}\right\|_{\beta_{1}}\right)\right) .
\end{aligned}
$$

Again,

$$
\begin{aligned}
& \Theta_{2}\left\|x_{2}, x_{3}, \cdots, x_{k}, x_{k+2}-x_{k+1}\right\|_{\beta_{2}} \\
& \quad \geq\left(\Gamma_{2}\left(b_{2}\right) \Theta_{2}\left(\left\|x_{1}, x_{2}, \cdots, x_{k-1}, x_{k+1}-x_{k}\right\|_{\beta_{2}}\right)\right), \\
& \Theta_{3}\left\|x_{2}, x_{3}, \cdots, x_{k}, x_{k+2}-x_{k+1}\right\|_{\beta_{3}} \\
& \quad \geq\left(\Gamma_{3}\left(b_{3}\right) \Theta_{3}\left(\left\|x_{1}, x_{2}, \cdots, x_{k-1}, x_{k+1}-x_{k}\right\|_{\beta_{3}}\right)\right) .
\end{aligned}
$$

Thus, we get

$$
\begin{aligned}
& \Theta_{1}\left(b_{1}\right) \leq \Gamma_{1}\left(b_{1}\right) \Theta_{1}\left(b_{1}\right) \Longrightarrow \Gamma_{1}\left(b_{1}\right) \geq 1, \\
& \Theta_{2}\left(b_{2}\right) \geq \Gamma_{2}\left(b_{2}\right) \Theta_{2}\left(b_{2}\right) \Longrightarrow \Gamma_{2}\left(b_{2}\right) \leq 1, \\
& \Theta_{3}\left(b_{3}\right) \geq \Gamma_{3}\left(b_{3}\right) \Theta_{3}\left(b_{3}\right) \Longrightarrow \Gamma_{3}\left(b_{3}\right) \leq 1,
\end{aligned}
$$

which is a contradiction; hence,

$$
\begin{aligned}
& \lim _{k \rightarrow \infty} M_{n}\left(\mathfrak{x}_{1}, \mathfrak{x}_{2}, \cdots, \mathfrak{x}_{k-1}, \mathfrak{x}_{k+1}-\mathfrak{x}_{k}, p\right)=1, \\
& \lim _{k \rightarrow \infty} A_{n}\left(\mathfrak{x}_{1}, \mathfrak{x}_{2}, \cdots, \mathfrak{x}_{k-1}, \mathfrak{x}_{k+1}-\mathfrak{x}_{k}, p\right)=0, \\
& \lim _{k \rightarrow \infty} N_{n}\left(\mathfrak{x}_{1}, \mathfrak{x}_{2}, \cdots, \mathfrak{x}_{k-1}, \mathfrak{x}_{k+1}-\mathfrak{x}_{k}, p\right)=0 .
\end{aligned}
$$

Suppose $p, \epsilon>0$. We have

$M_{n}\left(x_{1}, x_{2}, \cdots, x_{k-1}, x_{K+1}-x_{K}, \frac{p}{2}-\Theta_{1}^{-1}\left(\Gamma_{1}\left(\frac{P}{2}\right) \Theta_{1}\left(\frac{P}{2}\right)\right)\right) \geq 1-\epsilon$.

Similarly, for abstinence and falsity grades, we have

$$
\begin{aligned}
& A_{n}\left(x_{1}, x_{2}, \cdots, x_{k-1}, x_{K+1}-x_{K}, \frac{p}{2}-\Theta_{2}^{-1}\left(\Gamma_{2}\left(\frac{P}{2}\right) \Theta_{2}\left(\frac{P}{2}\right)\right)\right)<\epsilon, \\
& N_{n}\left(x_{1}, x_{2}, \cdots, x_{k-1}, x_{K+1}-x_{K}, \frac{p}{2}-\Theta_{3}^{-1}\left(\Gamma_{3}\left(\frac{P}{2}\right) \Theta_{3}\left(\frac{P}{2}\right)\right)\right)<\epsilon .
\end{aligned}
$$

By using the above analysis, we get, if $M_{n}\left(x_{1}, x_{2}, \cdots\right.$, $\left.\mathfrak{x}_{k-1}, \mathfrak{x}-\mathfrak{x}_{K}, p / 2\right) \geq 1-\epsilon$ and $A_{n}\left(\mathfrak{x}_{1}, \mathfrak{x}_{2}, \cdots, \mathfrak{x}_{k-1}, \mathfrak{x}-\mathfrak{x}_{K}, p / 2\right)$, $N_{n}\left(x_{1}, x_{2}, \cdots, x_{k-1}, x-x_{K}, p / 2\right)<\epsilon$, then

$$
\begin{aligned}
& M_{n}\left(x_{1}, x_{2}, \cdots, x_{k-1}, T(x)-x_{K}, \frac{p}{2}\right) \\
& \geq \min \left(M _ { n } \left(x_{1}, x_{2}, \cdots, x_{k-1}, T(x)-T\left(x_{K}\right), \frac{p}{2}\right.\right. \\
& \left.\left.\quad-\Theta_{1}^{-1}\left(\Gamma_{1}\left(\frac{P}{2}\right) \Theta_{1}\left(\frac{P}{2}\right)\right)\right)\right),
\end{aligned}
$$

$$
\begin{aligned}
& M_{n}\left(x_{1}, x_{2}, \cdots, x_{k-1}, T(x)-x_{K}, \Theta_{1}^{-1}\left(\Gamma_{1}\left(\frac{P}{2}\right) \Theta_{1}\left(\frac{P}{2}\right)\right)\right) \\
& \quad \geq \min \left(M_{n}\left(x_{1}, x_{2}, \cdots, x_{k-1}, x-x_{K}, \frac{P}{2}\right)\right), \\
& M_{n}\left(x_{1}, x_{2}, \cdots, x_{k-1}, x_{K+1}-x_{K}, \Theta_{1}^{-1}\left(\Gamma_{1}\left(\frac{P}{2}\right) \Theta_{1}\left(\frac{P}{2}\right)\right)\right) \geq 1-\epsilon .
\end{aligned}
$$

Similarly, resolving the grades of abstinence and falsity, we have

$$
\begin{aligned}
& A_{n}\left(x_{1}, x_{2}, \cdots, x_{k-1}, x_{K+1}-x_{K}, \Theta_{2}^{-1}\left(\Gamma_{2}\left(\frac{P}{2}\right) \Theta_{2}\left(\frac{P}{2}\right)\right)\right)<\epsilon, \\
& N_{n}\left(x_{1}, x_{2}, \cdots, x_{k-1}, x_{K+1}-x_{K}, \Theta_{3}^{-1}\left(\Gamma_{3}\left(\frac{P}{2}\right) \Theta_{3}\left(\frac{P}{2}\right)\right)\right)<\epsilon .
\end{aligned}
$$

Therefore,

$$
M_{n}\left(x_{1}, x_{2}, \cdots, x_{k-1}, \mathfrak{x}_{k}-\mathfrak{x}_{K}, \frac{p}{2}\right) \geq 1-\epsilon .
$$

Also, we note

$$
\begin{aligned}
& A_{n}\left(x_{1}, x_{2}, \cdots, x_{k-1}, x_{k}-x_{K}, \frac{p}{2}\right)<\epsilon, \\
& N_{n}\left(x_{1}, x_{2}, \cdots, x_{k-1}, x_{k}-x_{K}, \frac{p}{2}\right)<\epsilon .
\end{aligned}
$$

Then, for all $k \geq \mathbb{N}_{\mathrm{Nn}}$,

$$
\begin{aligned}
& M_{n}\left(x_{1}, x_{2}, \cdots, x_{k-1}, x_{k}-x_{Y}, p\right) \\
& \quad \geq \min \left\{M_{n}\left(x_{1}, x_{2}, \cdots, x_{k-1}, x_{k}-x_{K}, p\right), M_{n}\right. \\
& \left.\quad \cdot\left(x_{1}, x_{2}, \cdots, x_{k-1}, x_{Y}-x_{K}, p\right)\right\} \geq 1-\epsilon, \\
& M_{n}\left(x_{1}, x_{2}, \cdots, x_{k-1}, x_{Y}-x_{K}, p\right) \geq 1-\epsilon .
\end{aligned}
$$

Further, we find

$$
\begin{aligned}
& A_{n}\left(x_{1}, x_{2}, \cdots, x_{k-1}, x_{k}-x_{\gamma}, p\right)<\epsilon, \\
& N_{n}\left(x_{1}, x_{2}, \cdots, x_{k-1}, x_{k}-x_{\gamma}, p\right)<\epsilon .
\end{aligned}
$$

Since $\epsilon$ is arbitrary and the sequence $\left\{x_{\mathfrak{f}}\right\}$ is Cauchy, hence they are convergent. Therefore, $\lim \left\{x_{\mathfrak{f}}\right\}=x$. 
Suppose $p, \epsilon>0$; then, there exists $k_{0} \in \mathbb{N}_{\mathrm{Nn}}$ such that

$$
M_{n}\left(x_{1}, x_{2}, \cdots, x_{k-1}, x-x_{k}, p-\Theta_{1}^{-1}\left(\Gamma_{1}(p) \Theta_{1}(p)\right)\right) \geq 1-\epsilon .
$$
have

Similarly, observing for abstinence and falsity grades, we

$$
\begin{aligned}
& A_{n}\left(x_{1}, x_{2}, \cdots, x_{k-1}, x_{k}-x, p-\Theta_{2}^{-1}\left(\Gamma_{2}(p) \Theta_{2}(p)\right)\right)<\epsilon, \\
& N_{n}\left(x_{1}, x_{2}, \cdots, x_{k-1}, x_{k}-x, p-\Theta_{3}^{-1}\left(\Gamma_{3}(p) \Theta_{3}(p)\right)\right)<\epsilon,
\end{aligned}
$$

for all $k \geq k_{0}$. Hence,

$$
\begin{aligned}
& M_{n}\left(x_{1}, x_{2}, \cdots, x_{k-1}, T(x)-x, p\right) \\
& \quad \geq \min \left(M_{n}\left(x_{1}, x_{2}, \cdots, x_{k-1}, T(x)-x_{k+1}, p-\Theta_{1}^{-1}\left(\Gamma_{1}(p) \Theta_{1}(p)\right)\right)\right), \\
& \quad M_{n}\left(x_{1}, x_{2}, \cdots, x_{k-1}, x_{k+1}-x, \Theta_{1}^{-1}\left(\Gamma_{1}(p) \Theta_{1}(p)\right)\right) \\
& \quad \geq \min \left(M_{n}\left(x_{1}, x_{2}, \cdots, x_{k-1}, x-x_{k}, p\right)\right) \\
& M_{n}\left(x_{1}, x_{2}, \cdots, x_{k-1}, x_{k+1}-x, \Theta_{1}^{-1}\left(\Gamma_{1}(p) \Theta_{1}(p)\right)\right) \geq 1-\epsilon \\
& A_{n}\left(x_{1}, x_{2}, \cdots, x_{k-1}, T(x)-x, p\right)<\epsilon \\
& N_{n}\left(x_{1}, x_{2}, \cdots, x_{k-1}, T(x)-x, p\right)<\epsilon
\end{aligned}
$$

for all $k \geq k_{0}$. Therefore,

$$
\begin{aligned}
& M_{n}\left(x_{1}, x_{2}, \cdots, x_{k-1}, T(x)-x, p\right)=1, \\
& A_{n}\left(x_{1}, x_{2}, \cdots, x_{k-1}, T(x)-x, p\right)=0, \\
& N_{n}\left(x_{1}, x_{2}, \cdots, x_{k-1}, T(x)-x x, p\right)=0,
\end{aligned}
$$

for all $p>0$. Hence, $T(x)=x$; that is, $T$ has a fixed point in $X_{\mathrm{UNI}}$. Next, we prove the uniqueness of the fixed point. For this, we suppose $y$ is another fixed point $T$ in $X_{\mathrm{UNI}}$; then,

$$
\begin{aligned}
M_{n}\left(x_{1}, x_{2}, \cdots, x_{k-1}, x-y, p\right) \geq & \min \left(M _ { n } \left(x_{1}, x_{2}, \cdots, x_{k-1}, T(x)\right.\right. \\
& \left.\left.-T(y), \Theta_{1}^{-1}\left(\Gamma_{1}(p) \Theta_{1}(p)\right)\right)\right) \\
= & M_{n}\left(x_{1}, x_{2}, \cdots, x_{k-1}, x-y, p\right), \\
A_{n}\left(x_{1}, x_{2}, \cdots, x_{k-1}, x-y, p\right)= & M_{n}\left(x_{1}, x_{2}, \cdots, x_{k-1}, x-y, p\right), \\
N_{n}\left(x_{1}, x_{2}, \cdots, x_{k-1}, x-y, p\right)= & M_{n}\left(x_{1}, x_{2}, \cdots, x_{k-1}, x-y, p\right) .
\end{aligned}
$$

Hence, $p=\Theta_{1}^{-1}\left(\Gamma_{1}(p) \Theta_{1}(p)\right) \Longrightarrow \Theta_{1}(p)=\Gamma_{1}(p) \Theta_{1}(p)$,

$$
\begin{aligned}
& p=\Theta_{2}^{-1}\left(\Gamma_{2}(p) \Theta_{2}(p)\right) \Longrightarrow \Theta_{2}(p)=\Gamma_{2}(p) \Theta_{2}(p), \\
& p=\Theta_{3}^{-1}\left(\Gamma_{3}(p) \Theta_{3}(p)\right) \Longrightarrow \Theta_{3}(p)=\Gamma_{3}(p) \Theta_{3}(p) .
\end{aligned}
$$

Therefore, $\Gamma_{1}(p)=1, \Gamma_{2}(p)=1$, and $\Gamma_{3}(p)=1$. It is a contradiction; thus, for all $k \in \mathbb{N}_{\mathrm{Nn}}$ and $k \longrightarrow \infty$, we obtain

$$
\begin{aligned}
& M_{n}\left(x_{1}, x_{2}, \cdots, x_{k-1}, x-\ddot{y}, p\right)=1, \\
& A_{n}\left(x_{1}, x_{2}, \cdots, x_{k-1}, x-y, p\right)=0, \\
& N_{n}\left(x_{1}, x_{2}, \cdots, x_{k-1}, x-y, p\right)=0,
\end{aligned}
$$

for all $p>0$. Hence, $x=\ddot{y}$. Hence, $T$ has a unique fixed point in $X_{\mathrm{UNI}}$.

Example 12. For a Banach space $\left(X_{\mathrm{UNI}},\|\|.\right)$, we define the decreasing mapping $\Gamma_{1}$ and increasing mappings $\Gamma_{2}$ and $\Gamma_{3}$, such that $\Gamma_{1}:(0,+\infty) \longrightarrow[0,1)$ and $\Gamma_{2}:(0,+\infty) \longrightarrow[0,1)$, $\Gamma_{3}:(0,+\infty) \longrightarrow[0,1)$, and $T: X_{\mathrm{UNI}} \longrightarrow X_{\mathrm{UNI}}$ are such that for all $x, y \in X_{\mathrm{UNI}}$,

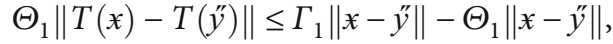

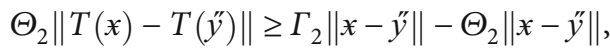

$$
\begin{aligned}
& \Theta_{3}\|T(x)-T(\ddot{y})\| \geq \Gamma_{3}\|x-y\|-\Theta_{3}\|x-y ̋\|,
\end{aligned}
$$

where $\Theta_{1} \in \bar{T}_{m-1}, \Theta_{2} \in \bar{T}_{a-2}$, and $\Theta_{3} \in \bar{T}_{n-3}$. Suppose that $\Gamma_{1}$ $\Theta_{1}$ is nondecreasing and $\Gamma_{2} \Theta_{2}, \Gamma_{3} \Theta_{3}$ are nonincreasing functions with

$$
\begin{aligned}
& \beta_{1}\left(\Theta_{1}^{-1}\left(\Gamma_{1}(p) \Theta_{1}(p)\right)\right) \leq \Theta_{1}^{-1}\left(\Gamma_{1}\left(\beta_{1} p\right) \Theta_{1}\left(\beta_{1} p\right)\right) \\
& \beta_{2}\left(\Theta_{2}^{-1}\left(\Gamma_{2}(p) \Theta_{2}(p)\right)\right) \geq \Theta_{2}^{-1}\left(\Gamma_{2}\left(\beta_{2} p\right) \Theta_{2}\left(\beta_{2} p\right)\right) \\
& \beta_{3}\left(\Theta_{3}^{-1}\left(\Gamma_{3}(p) \Theta_{3}(p)\right)\right) \geq \Theta_{3}^{-1}\left(\Gamma_{3}\left(\beta_{3} p\right) \Theta_{3}\left(\beta_{3} p\right)\right)
\end{aligned}
$$

for all $p \in[0,+\infty), \beta_{1}, \beta_{2}, \beta_{3} \in[0,1]$. Further, define picture fuzzy $n$-norm $M_{n}, A_{n}, N_{n}$ as in Example 10. Consider that

$$
\begin{aligned}
& M_{n}\left(x_{1}, x_{2}, \cdots, x_{k-1}, x-\ddot{y}, p\right) \geq \alpha, \\
& A_{n}\left(x_{1}, x_{2}, \cdots, x_{k-1}, x-y, p\right)<1-\alpha, \\
& N_{n}\left(x_{1}, x_{2}, \cdots, x_{k-1}, x-y, p\right)<1-\alpha .
\end{aligned}
$$

By using the three cases of Example 10 and using Theorem 11, we explore that the function $T$ has a unique fixed point in $X_{\mathrm{UNI}}$. Hence, the solution is completed. Further, we have utilized more results based on $B_{\mathrm{PF}}$ to show the proficiency of the proven approaches.

Theorem 13. Let $B_{P F}\left(X_{U N I}, M_{m}, A_{a}, N_{n}, *_{c t},{ }_{c t c}\right)$. Let the grade of truth, abstinence, and falsity satisfy the conditions of Definition 4. Now, we define the mapping $T: X_{U N I} \longrightarrow$ $X_{U N I}$, such that

$$
\begin{aligned}
M_{n}\left(x_{1}, x_{2}, \cdots, x_{k-1}, x-y, p\right) \geq \alpha & \Longrightarrow M_{n}\left(x_{1}, x_{2}, \cdots, x_{k-1}, T(x)\right. \\
& \left.-T(\ddot{y}), \Theta_{1}^{-1}\left(\Theta_{1}(P)-\Theta_{1}^{\prime}(P)\right)\right) \geq \alpha
\end{aligned}
$$




$$
\begin{aligned}
A_{n}\left(\mathfrak{x}_{1}, \mathfrak{x}_{2}, \cdots, \mathfrak{x}_{k-1}, \mathfrak{x}-\ddot{y}, p\right)< & 1-\alpha \Longrightarrow A_{n}\left(\mathfrak{x}_{1}, \mathfrak{x}_{2}, \cdots, \mathfrak{x}_{k-1}, T(\mathfrak{x})\right. \\
& \left.-T(\ddot{y}), \Theta_{2}^{-1}\left(\Theta_{2}(P)-\Theta_{2}^{\prime}(P)\right)\right) \\
< & 1-\alpha \\
N_{n}\left(x_{1}, x_{2}, \cdots, x_{k-1}, \mathfrak{x}-\ddot{y}, p\right)< & 1-\alpha \Longrightarrow N_{n}\left(\mathfrak{x}_{1}, \mathfrak{x}_{2}, \cdots, \mathfrak{x}_{k-1}, T(\mathfrak{x})\right. \\
& \left.-T(\ddot{y}), \Theta_{3}^{-1}\left(\Theta_{3}(P)-\Theta_{3}^{\prime}(P)\right)\right) \\
< & 1-\alpha\},
\end{aligned}
$$

where $\left(\Theta_{1}(P), \Theta_{1}^{\prime}(P)\right) \in \bar{T}_{m-1}, \quad\left(\Theta_{2}(P), \Theta_{2}^{\prime}(P)\right) \in \bar{T}_{a-2}$, and $\left(\Theta_{3}(P)-\Theta_{3}^{\prime}(P)\right) \in \bar{T}_{n-3}$, for all $x_{1}, x_{2}, \cdots, x_{k-1} \in X_{U N I}, x, y \in$ $X_{U N I}, p>0$ with $\alpha \in(0,1]$ and $\left(\Theta_{1}(P) \geq \Theta_{1}^{\prime}(P)\right),\left(\Theta_{2}(P) \geq\right.$ $\left.\Theta_{2}^{\prime}(P)\right),\left(\Theta_{3}(P) \geq \Theta_{3}^{\prime}(P)\right)$. Then, $T$ has a unique fixed point in $\mathrm{X}_{U N I}$.

Proof. Let $x_{0} \in X_{\mathrm{UNI}}$ with $x_{k+1}=T\left(x_{k}\right) \forall k \in \mathbb{N}_{\mathrm{Nn}}$. By using Remark 7 and equation (66), we get

$$
\begin{aligned}
& M_{n}\left(x_{1}, x_{2}, \cdots, x_{k-1}, x-\ddot{y}, p\right) \\
& \leq M_{n}\left(x_{1}, x_{2}, \cdots, x_{k-1}, T(x)-T(\ddot{y}), \Theta_{1}^{-1}\left(\Theta_{1}(P)-\Theta_{1}^{\prime}(P)\right)\right), \\
& A_{n}\left(x_{1}, x_{2}, \cdots, x_{k-1}, x-\ddot{y}, p\right) \\
& \geq A_{n}\left(x_{1}, x_{2}, \cdots, x_{k-1}, T(x)-T(y), \Theta_{2}^{-1}\left(\Theta_{2}(P)-\Theta_{2}^{\prime}(P)\right)\right), \\
& N_{n}\left(x_{1}, x_{2}, \cdots, x_{k-1}, \mathfrak{x}-\ddot{y}, p\right) \\
& \geq N_{n}\left(x_{1}, x_{2}, \cdots, x_{k-1}, T(x)-T(y), \Theta_{3}^{-1}\left(\Theta_{3}(P)-\Theta_{3}^{\prime}(P)\right)\right) .
\end{aligned}
$$

Further, from the above equations, we obtain

$$
\begin{aligned}
& M_{n}\left(x_{1}, x_{2}, \cdots, x_{k-1}, x_{k+1}-x_{k}, p\right) \\
& \quad \leq M_{n}\left(x_{2}, x_{3}, \cdots, x_{k}, x_{k+2}-x_{k+1}, \Theta_{1}^{-1}\left(\Theta_{1}(P)-\Theta_{1}^{\prime}(P)\right)\right) \\
& \quad \leq M_{n}\left(x_{2}, x_{3}, \cdots, x_{k}, x_{k+2}-x_{k+1}, p\right), \\
& A_{n}\left(x_{1}, x_{2}, \cdots, x_{k-1}, x_{k+1}-x_{k}, p\right) \\
& \quad \geq A_{n}\left(x_{2}, x_{3}, \cdots, x_{k}, x_{k+2}-x_{k+1}, \Theta_{2}^{-1}\left(\Theta_{2}(P)-\Theta_{2}^{\prime}(P)\right)\right) \\
& \quad \geq A_{n}\left(x_{2}, x_{3}, \cdots, x_{k}, x_{k+2}-x_{k+1}, p\right), \\
& N_{n}\left(x_{1}, x_{2}, \cdots, x_{k-1}, x_{k+1}-x_{k}, p\right) \\
& \quad \geq N_{n}\left(x_{2}, x_{3}, \cdots, x_{k}, x_{k+2}-x_{k+1}, \Theta_{3}^{-1}\left(\Theta_{3}(P)-\Theta_{3}^{\prime}(P)\right)\right) \\
& \quad \leq N_{n}\left(x_{2}, x_{3}, \cdots, x_{k}, x_{k+2}-x_{k+1}, p\right) .
\end{aligned}
$$

It is clear from the above analysis that $\left\{M_{n}\left(x_{1}, x_{2}\right.\right.$, $\left.\left.\cdots, x_{k-1}, x_{k+1}-x_{k}, p\right)\right\}$ is a bounded nondecreasing sequence and the sequences $\left\{A_{n}\left(x_{1}, x_{2}, \cdots, x_{k-1}, x_{k+1}-x_{k}, p\right)\right\}$ and $\left\{N_{n}\left(x_{1}, x_{2}, \cdots, x_{k-1}, x_{k+1}-x_{k}, p\right)\right\}$ are bounded and nonincreasing. Then, the limit of these equations exists. We suppose that

$$
\begin{aligned}
& \lim _{k \rightarrow \infty} M_{n}\left(x_{1}, x_{2}, \cdots, x_{k-1}, x_{k+1}-x_{k}, p\right)<\beta_{1}<1, \\
& \lim _{k \rightarrow \infty} A_{n}\left(x_{1}, x_{2}, \cdots, x_{k-1}, x_{k+1}-x_{k}, p\right)>\beta_{2}>1, \\
& \lim _{k \rightarrow \infty} N_{n}\left(x_{1}, x_{2}, \cdots, x_{k-1}, x_{k+1}-x_{k}, p\right)>\beta_{3}>1 .
\end{aligned}
$$

Therefore, we have

$$
\begin{aligned}
& M_{n}\left(x_{2}, x_{3}, \cdots, x_{k}, x_{k+2}-x_{k+1}, q\right) \geq M_{n}\left(x_{1}, x_{2}, \cdots, x_{k-1}, x_{k+1}-x_{k}, q\right), \\
& A_{n}\left(x_{2}, x_{3}, \cdots, x_{k}, x_{k+2}-x_{k+1}, q\right) \leq A_{n}\left(x_{1}, x_{2}, \cdots, x_{k-1}, x_{k+1}-x_{k}, q\right), \\
& N_{n}\left(x_{2}, x_{3}, \cdots, x_{k}, x_{k+2}-x_{k+1}, q\right) \leq N_{n}\left(x_{1}, x_{2}, \cdots, x_{k-1}, x_{k+1}-x_{k}, q\right), \\
& 0<p \leq\left\|x_{2}, x_{3}, \cdots, x_{k}, x_{k+2}-x_{k+1}\right\|_{\beta_{1}} \leq\left\|x_{2}, x_{3}, \cdots, x_{k-1}, x_{k+1}-x_{k}\right\|_{\beta_{1}}, \\
& 0<p \leq\left\|x_{2}, x_{3}, \cdots, x_{k-1}, x_{k+1}-x_{k}\right\|_{\beta_{2}} \leq\left\|x_{2}, x_{3}, \cdots, x_{k}, x_{k+2}-x_{k+1}\right\|_{\beta_{2}}, \\
& 0<p \leq\left\|x_{2}, x_{3}, \cdots, x_{k-1}, x_{k+1}-x_{k}\right\|_{\beta_{3}} \leq\left\|x_{2}, x_{3}, \cdots, x_{k}, x_{k+2}-x_{k+1}\right\|_{\beta_{3}} .
\end{aligned}
$$

Then, the limit of these equations also exists. We have

$$
\begin{aligned}
& \lim _{k \rightarrow \infty}\left\|x_{1}, x_{2}, \cdots, x_{k-1}, x_{k+1}-x_{k}\right\|_{\beta_{1}}=b_{1}, \\
& \lim _{k \rightarrow \infty}\left\|x_{1}, x_{2}, \cdots, x_{k-1}, x_{k+1}-x_{k}\right\|_{\beta_{2}}=b_{2}, \\
& \lim _{k \rightarrow \infty}\left\|x_{1}, x_{2}, \cdots, x_{k-1}, x_{k+1}-x_{k}\right\|_{\beta_{3}}=b_{3} .
\end{aligned}
$$

If $M_{n}\left(x_{2}, x_{3}, \cdots, x_{k}, x_{k+2}-x_{k+1}, q\right) \geq \beta_{1}, A_{n}\left(x_{2}, x_{3}, \cdots, x_{k}\right.$, $\left.x_{k+2}-x_{k+1}, q\right)<\beta_{2}$, and $N_{n}\left(x_{2}, x_{3}, \cdots, x_{k}, x_{k+2}-x_{k+1}, q\right)<\beta_{3}$, then

$$
\begin{aligned}
& M_{n}\left(x_{2}, x_{3}, \cdots, x_{k}, x_{k+2}-x_{k+1}, \Theta_{1}^{-1}\left(\Theta_{1}(P)-\Theta_{1}^{\prime}(P)\right)\right) \\
& \quad \geq M_{n}\left(x_{1}, x_{2}, \cdots, x_{k-1}, x_{k+1}-x_{k}, q\right) \geq \beta_{1}, \\
& A_{n}\left(x_{2}, x_{3}, \cdots, x_{k}, x_{k+2}-x_{k+1}, \Theta_{2}^{-1}\left(\Theta_{2}(P)-\Theta_{2}^{\prime}(P)\right)\right) \\
& \quad \leq A_{n}\left(x_{1}, x_{2}, \cdots, x_{k-1}, x_{k+1}-x_{k}, q\right)<\beta_{2}, \\
& M_{n}\left(x_{2}, x_{3}, \cdots, x_{k}, x_{k+2}-x_{k+1}, \Theta_{3}^{-1}\left(\Theta_{3}(P)-\Theta_{3}^{\prime}(P)\right)\right) \\
& \quad \leq M_{n}\left(x_{1}, x_{2}, \cdots, x_{k-1}, x_{k+1}-x_{k}, q\right)<\beta_{3} .
\end{aligned}
$$

Therefore,

$$
\begin{aligned}
\left\|x_{2}, x_{3}, \cdots, x_{k}, x_{k+2}-x_{k+1}\right\|_{\beta_{1}} \leq \Theta_{1}^{-1}\left(\Theta_{1}(P)-\Theta_{1}^{\prime}(P)\right) \\
\Theta_{1}\left\|x_{2}, x_{3}, \cdots, x_{k}, x_{k+2}-x_{k+1}\right\|_{\beta_{1}} \leq\left(\Theta_{1}(P)-\Theta_{1}^{\prime}(P)\right) .
\end{aligned}
$$

Similarly, we can find

$$
\begin{aligned}
& \Theta_{2}\left\|x_{2}, x_{3}, \cdots, x_{k}, x_{k+2}-x_{k+1}\right\|_{\beta_{2}} \geq\left(\Theta_{2}(P)-\Theta_{2}^{\prime}(P)\right), \\
& \Theta_{3}\left\|x_{2}, x_{3}, \cdots, x_{k}, x_{k+2}-x_{k+1}\right\|_{\beta_{3}} \geq\left(\Theta_{3}(P)-\Theta_{3}^{\prime}(P)\right) .
\end{aligned}
$$


Clearly, $p \longrightarrow\left\|x_{1}, x_{2}, \cdots, x_{k-1}, x_{k+1}-x_{k}\right\|_{\beta_{1}}, \| x_{1}, x_{2}, \cdots$, $x_{k-1}, x_{k+1}-x_{k}\left\|_{\beta_{2}},\right\| x_{1}, x_{2}, \cdots, x_{k-1}, x_{k+1}-x_{k} \|_{\beta_{3}}$; hence,

$$
\begin{aligned}
\Theta_{1}\left\|x_{2}, x_{3}, \cdots, x_{k}, x_{k+2}-x_{k+1}\right\|_{\beta_{1}} \leq & \left(\Theta_{1}\left(\left\|x_{1}, x_{2}, \cdots, x_{k-1}, x_{k+1}-x_{k}\right\|_{\beta_{1}}\right)\right. \\
& \left.-\Theta_{1}^{\prime}\left(\left\|x_{1}, x_{2}, \cdots, x_{k-1}, x_{k+1}-x_{k}\right\|_{\beta_{1}}\right)\right) .
\end{aligned}
$$

Also, we write

$$
\begin{aligned}
\Theta_{2}\left\|x_{2}, x_{3}, \cdots, x_{k}, x_{k+2}-x_{k+1}\right\|_{\beta_{2}} \geq & \left(\Theta_{2}\left(\left\|x_{1}, x_{2}, \cdots, x_{k-1}, x_{k+1}-x_{k}\right\|_{\beta_{1}}\right)\right. \\
& \left.-\Theta_{2}^{\prime}\left(\left\|x_{1}, x_{2}, \cdots, x_{k-1}, x_{k+1}-x_{k}\right\|_{\beta_{1}}\right)\right), \\
\Theta_{3}\left\|x_{2}, x_{3}, \cdots, x_{k}, x_{k+2}-x_{k+1}\right\|_{\beta_{3}} \geq & \left(\Theta_{3}\left(\left\|x_{1}, x_{2}, \cdots, x_{k-1}, x_{k+1}-x_{k}\right\|_{\beta_{1}}\right)\right. \\
& \left.-\Theta_{3}^{\prime}\left(\left\|x_{1}, x_{2}, \cdots, x_{k-1}, x_{k+1}-x_{k}\right\|_{\beta_{1}}\right)\right) .
\end{aligned}
$$

Thus, we get

$$
0 \leq \Theta_{1}(p) \leq \Theta_{1}\left(b_{1}\right) \leq \Theta_{1}\left(b_{1}\right)-\Theta_{1}^{\prime}\left(b_{1}\right) \leq \Theta_{1}\left(b_{1}\right) .
$$

Similarly,

$$
\begin{aligned}
& \Theta_{2}(p) \geq \Theta_{2}\left(b_{2}\right) \geq \Theta_{2}\left(b_{2}\right)-\Theta_{2}^{\prime}\left(b_{2}\right) \geq \Theta_{2}\left(b_{2}\right), \\
& \Theta_{3}(p) \geq \Theta_{3}\left(b_{3}\right) \geq \Theta_{3}\left(b_{3}\right)-\Theta_{3}^{\prime}\left(b_{3}\right) \geq \Theta_{3}\left(b_{3}\right) .
\end{aligned}
$$

It is a contradiction; hence,

$$
\begin{aligned}
& \lim _{k \rightarrow \infty} M_{n}\left(x_{1}, x_{2}, \cdots, x_{k-1}, x_{k+1}-x_{k}, p\right)=1, \\
& \lim _{k \rightarrow \infty} A_{n}\left(x_{1}, x_{2}, \cdots, x_{k-1}, x_{k+1}-x_{k}, p\right)=0, \\
& \lim _{k \rightarrow \infty} N_{n}\left(x_{1}, x_{2}, \cdots, x_{k-1}, x_{k+1}-x_{k}, p\right)=0 .
\end{aligned}
$$

The rest of the proof to express for all $p>0$ the equation $T(x)=x$ can be obtained using the similar technique of Theorem 9 and Theorem 11; that is, $T$ has a fixed point in $X_{\mathrm{UNI}}$. Next, we prove the uniqueness of the fixed point. For this, we suppose $y$ is another fixed point $T$ in $X_{\mathrm{UNI}}$; then,

$$
\begin{aligned}
& M_{n}\left(x_{1}, x_{2}, \cdots, x_{k-1}, x-\ddot{y}, p\right) \\
& \quad \geq \min \left(M_{n}\left(x_{1}, x_{2}, \cdots, x_{k-1}, T(x)-T(\ddot{y}), \Theta_{1}^{-1}\left(\Theta_{1}(P)-\Theta_{1}^{\prime}(P)\right)\right)\right) \\
& \quad=M_{n}\left(x_{1}, x_{2}, \cdots, x_{k-1}, x-\ddot{y}, p\right), \\
& A_{n}\left(x_{1}, x_{2}, \cdots, x_{k-1}, x-\ddot{y}, p\right)=M_{n}\left(x_{1}, x_{2}, \cdots, x_{k-1}, x-\ddot{y}, p\right), \\
& N_{n}\left(x_{1}, x_{2}, \cdots, x_{k-1}, x-y, p\right)=M_{n}\left(x_{1}, x_{2}, \cdots, x_{k-1}, x-\ddot{y}, p\right) .
\end{aligned}
$$
$\Theta_{1}^{\prime}(P)$,

$$
\begin{aligned}
& p=\Theta_{2}^{-1}\left(\Theta_{2}(P)-\Theta_{2}^{\prime}(P)\right) \Longrightarrow \Theta_{2}(p)=\Theta_{2}(P)-\Theta_{2}^{\prime}(P), \\
& p=\Theta_{3}^{-1}\left(\Theta_{3}(P)-\Theta_{3}^{\prime}(P)\right) \Longrightarrow \Theta_{3}(p)=\Theta_{3}(P)-\Theta_{3}^{\prime}(P) .
\end{aligned}
$$

Therefore, $\Theta_{1}^{\prime}(p)=1, \Theta_{2}^{\prime}(p)=1$, and $\Theta_{3}^{\prime}(p)=1$. It is a contradiction; thus, as $k \longrightarrow \infty$, we get

$$
\begin{aligned}
& M_{n}\left(x_{1}, x_{2}, \cdots, x_{k-1}, x-y, p\right)=1, \\
& A_{n}\left(x_{1}, x_{2}, \cdots, x_{k-1}, x-y, p\right)=0, \\
& N_{n}\left(x_{1}, x_{2}, \cdots, x_{k-1}, x-y, p\right)=0,
\end{aligned}
$$

for all $p>0$. Hence, $x=y$. Thus, $T$ has a unique fixed point in $X_{\mathrm{UNI}}$.

Example 14. For a Banach space $\left(X_{\mathrm{UNI}},\|\cdot\|\right)$, we define the mapping $T: X_{\mathrm{UNI}} \longrightarrow X_{\mathrm{UNI}}$ such that for all $x, y \in X_{\mathrm{UNI}}$,

$$
\begin{aligned}
& \Theta_{1}\|T(x)-T(y)\| \leq \Theta_{1}\|x-\ddot{y}\|-\Theta_{1}^{\prime}\|x-y=\|, \\
& \Theta_{2}\|T(x)-T(y)\| \geq \Theta_{2}\|x-\ddot{y}\|-\Theta_{2}^{\prime}\|x-\ddot{y}\| \text {, } \\
& \Theta_{3}\|T(x)-T(y)\| \geq \Theta_{3}\|x-\ddot{y}\|-\Theta_{3}^{\prime} \| x-y=\mid
\end{aligned}
$$

where $\Theta_{1}, \Theta_{1}^{\prime} \in \bar{T}_{m-1}, \Theta_{2}, \Theta_{2}^{\prime} \in \bar{T}_{a-2}$, and $\Theta_{3}, \Theta_{3}^{\prime} \in \bar{T}_{n-3}$. Suppose $\Theta_{1}-\Theta_{1}^{\prime}$ is nondecreasing and $\Theta_{2}-\Theta_{2}^{\prime}, \Theta_{3}-\Theta_{3}^{\prime}$ are nonincreasing functions with

$$
\begin{array}{r}
\beta_{1}\left(\Theta_{1}^{-1}\left(\Theta_{1}-\Theta_{1}^{\prime}\right)\right) \leq \Theta_{1}^{-1}\left(\Theta_{1}\left(\beta_{1} p\right)-\Theta_{1}^{\prime}\left(\beta_{1} p\right)\right), \\
\beta_{2}\left(\Theta_{2}^{-1}\left(\Gamma_{2}(p) \Theta_{2}(p)\right)\right) \geq \Theta_{2}^{-1}\left(\Theta_{2}\left(\beta_{2} p\right)-\Theta_{2}^{\prime}\left(\beta_{2} p\right)\right), \\
\beta_{3}\left(\Theta_{3}^{-1}\left(\Gamma_{3}(p) \Theta_{3}(p)\right)\right) \geq \Theta_{3}^{-1}\left(\Theta_{3}\left(\beta_{3} p\right)-\Theta_{3}^{\prime}\left(\beta_{3} p\right)\right),
\end{array}
$$

for all $p \in[0,+\infty)$ and $\beta_{1}, \beta_{2}, \beta_{3} \in[0,1]$. Further, define picture fuzzy $n$-norm $M_{n}, A_{n}, N_{n}$ as in Example 10. Consider that

$$
\begin{aligned}
& M_{n}\left(x_{1}, x_{2}, \cdots, x_{k-1}, x-\ddot{y}, p\right) \geq \alpha, \\
& A_{n}\left(x_{1}, x_{2}, \cdots, x_{k-1}, x-\ddot{y}, p\right)<1-\alpha, \\
& N_{n}\left(x_{1}, x_{2}, \cdots, x_{k-1}, x-\ddot{y}, p\right)<1-\alpha .
\end{aligned}
$$

We explore that the function $T$ has a unique fixed point in $X_{\mathrm{UNI}}$. Hence, the solution is completed.

\section{Conclusion}

A picture fuzzy set is more proficient and more capable than an intuitionistic fuzzy set and fuzzy to cope with uncertain 
and unpredictable information in realistic issues. Keeping the advantages of the picture fuzzy set and a $n$-norm linear space, the manuscript made the following advancements in the existing literature:

(1) The novel picture fuzzy $n$-norm linear space and its basic properties are explored

(2) Some novel contractive conditions based on $N_{\mathrm{PF}}$ are presented. By using these contractive conditions, we have explored some fixed point theorems for a picture fuzzy $n$-Banach space $\left(B_{\mathrm{PF}}\right)$. It was observed that these results are more modified and more general than the existing ones in the literature, which are based on intuitionistic fuzzy $n$-Banach spaces $\left(B_{\mathrm{IF}}\right)$ and fuzzy $n$-Banach spaces

(3) The reliability and effectiveness of the obtained main theorems are expressed, and several examples are presented afterwards

\section{Data Availability}

The data used to support the findings of this study are available from the corresponding author upon request.

\section{Conflicts of Interest}

We declare that we do not have any commercial or associative interests that represent conflicts of interest in connection with this manuscript. There are no professional or other personal interests that can inappropriately influence our submitted work.

\section{Authors' Contributions}

All authors contributed equally to the writing of this article. All authors read and approved the final manuscript.

\section{References}

[1] W. Sintunavarat, S. Chauhan, and P. Kumam, "Some fixed point results in modified intuitionistic fuzzy metric spaces," Afrika Matematika, vol. 25, no. 2, pp. 461-473, 2014.

[2] R. Saadati, S. Sedghi, and N. Shobe, "Modified intuitionistic fuzzy metric spaces and some fixed point theorems," Chaos, Solitons \& Fractals, vol. 38, no. 1, pp. 36-47, 2008.

[3] W. Walter, "An elementary proof of the Cauchy-Kowalevsky theorem," The American Mathematical Monthly, vol. 92, no. 2, pp. 115-126, 2018.

[4] C. H. Wagner, "A generic approach to iterative methods," Mathematics Magazine, vol. 55, no. 5, pp. 259-273, 2018.

[5] M. Freeman, "The inverse as a fixed point in function space," The American Mathematical Monthly, vol. 83, no. 5, pp. 344-348, 2018.

[6] L. A. Zadeh, "Fuzzy sets," Information and Control, vol. 8, no. 3, pp. 338-353, 1965.

[7] K. T. Atanassov, "Intuitionistic fuzzy sets," in Intuitionistic Fuzzy Sets, pp. 1-137, Physica, Heidelberg, 1999.

[8] B. C. Cường, "Picture fuzzy sets," Journal of Computer Science and Cybernetics, vol. 30, no. 4, pp. 409-420, 2015.
[9] N. Konwar and P. Debnath, "Some new contractive conditions and related fixed point theorems in intuitionistic fuzzy nBanach spaces," Journal of Intelligent \& Fuzzy Systems, vol. 34, no. 1, pp. 361-372, 2018.

[10] W. Kumam, P. Sukprasert, P. Kumam, A. Shoaib, A. Shahzad, and Q. Mahmood, "Some fuzzy fixed point results for fuzzy mappings in complete b-metric spaces," Cogent Mathematics \& Statistics, vol. 5, no. 1, pp. 1458933-1498952, 2018.

[11] C. K. B. Ampadu, "Some fixed point theory results for convex contraction mapping of order 2," JP Journal of Fixed Point Theory and Applications, vol. 12, no. 2-3, pp. 81-130, 2017.

[12] C. Ampadu and A. H. Ansari, "Fixed point theorems in complete multiplicative metric spaces with application to multiplicative analogue of C-class functions," JP Journal of Fixed Point Theory and Applications, vol. 11, no. 2, pp. 113-124, 2016.

[13] E. Ameer, H. Aydi, M. Arshad, and M. de la Sen, "Hybrid Ćirić type graphic $Y, \Lambda$-contraction mappings with applications to electric circuit and fractional differential equations," Symmetry, vol. 12, no. 3, p. 467, 2020.

[14] H. Qawaqneh, M. Md Noorani, W. Shatanawi, H. Aydi, and H. Alsamir, "Fixed point results for multi-valued contractions in b-metric spaces and an application," Mathematics, vol. 7, no. 2, p. 132, 2019.

[15] N. Alamgir, Q. Kiran, H. Isik, and H. Aydi, "Fixed point results via a Hausdorff controlled type metric," Advances in Difference Equations, vol. 2020, no. 1, Article ID 24, 2020.

[16] H. Afshari, H. Aydi, and E. Karapınar, "On generalized $\alpha-\psi$ Geraghty contractions on b-metric spaces," Georgian Mathematical Journal, vol. 27, no. 1, pp. 9-21, 2020. 